\title{
Prognosis of COVID-19 Pneumonia Can Be Early Predicted Combining Age-Adjusted Charlson Comorbidity Index, CRB Score and Baseline Oxygen Saturation.
}

Pilar Nuevo-Ortega ( $\nabla$ pilarnuevoortega@gmail.com )

Hospital Universitario Virgen de la Victoria https://orcid.org/0000-0002-4600-4045

\section{Carmen Reina-Artacho}

Hospital Universitario Virgen de la Victoria

Francisco Dominguez-Moreno

Hospital Universitario Virgen de la Victoria

Victor Manuel Becerra-Muñoz

Hospital Universitario Virgen de la Victoria

Luis Ruiz-Del-Fresno

Hospital Universitario Virgen de la Victoria

Maria Antonia Estecha-Foncea

Hospital Universitario Virgen de la Victoria

\section{Research}

Keywords: Coronavirus disease 2019, Disease severity, Mortality risk score, Charlson Comorbidity Index, CRB score, Logistics model, Pulse oximetry.

Posted Date: March 30th, 2021

DOI: https://doi.org/10.21203/rs.3.rs-209680/v2

License: (c) (i) This work is licensed under a Creative Commons Attribution 4.0 International License. Read Full License

Version of Record: A version of this preprint was published at Scientific Reports on February 11th, 2022. See the published version at https://doi.org/10.1038/s41598-022-06199-3. 


\section{Abstract}

Background: In potentially severe diseases in general and COVID-19 in particular, it is vital to early identify those patients who are going to develop complications. The last update of a recent living systematic review dedicated to predictive models in COVID-19,[1] critically appraises 145 models, 8 of them focused on prediction of severe disease and 23 on mortality. Unfortunately, in all 145 models, they found a risk of bias significant enough to finally "not recommend any for clinical use". Authors suggest concentrating on avoiding biases in sampling and prioritising the study of already identified predictive factors, rather than the identification of new ones that are often dependent on the database. Our objective is to develop a model to predict which patients with COVID-19 pneumonia are at high risk of developing severe illness or dying, using basic and validated clinical tools.

Methods: prospective cohort of consecutive patients admitted in a teaching hospital during the "first wave" of the COVID-19 pandemic. Follow-up to discharge from hospital. Multiple logistic regression selecting variables according to clinical and statistical criteria.

Results: 404 consecutive patients were evaluated, 392 (97\%) completed follow-up. Mean age was 61 years; $59 \%$ were men. The median burden of comorbidity was 2 points in the Age-adjusted Charlson Comorbidity Index, CRB was abnormal in $18 \%$ of patients and basal oxygen saturation on admission lower than $90 \%$ in $18 \%$. A model composed of Age-adjusted Charlson Comorbidity Index, CRB score and basal oxygen saturation can predict unfavorable evolution or death with an area under the ROC curve of 0.85 (95\% Cl: $0.80-0.89)$, and 0.90 (95\% $\mathrm{Cl}$ : 0.86 to 0.94$)$, respectively.

Conclusion: prognosis of COVID-19 pneumonia can be predicted in the out-of-hospital environment using two classic prognostic scales and a pocket pulse oximeter.

\section{Introduction}

In potentially severe diseases in general, and COVID-19 in particular, it is vital to early identify those patients who are going to develop complications. Physicians often care for patients who present a clearly mild or severe profile and do not need to calculate a predictive index to make decisions. However, in other cases, the path to follow is not so clear, and that is when a prediction rule can be helpful, e.g., in a patient with mild to moderate acute symptoms, but with a weak baseline situation; or in a patient who does have striking acute symptoms but is young and healthy. It can also be helpful for healthcare managers when need to quantify the healthcare demand that it is going to be faced and prepare the necessary resources in advance. Finally, a suitable predictive rule would be useful as a quality control tool for both, clinical physicians, and healthcare managers.

Motivated by the urgent need to characterise COVID-19 there has been a blast of publications (more than 80,000 up to early December 2020); the symptoms and initial characteristics of the disease are well known, but the determinants of its course are less clear. A living systematic review dedicated to predictive models in COVID-19,[1] in its latest version (search updated May 5), has found 145 models, 8 of them focused on prediction of severe disease and 23 on mortality. Unfortunately, in all 145 models, they found a risk of bias significant enough to finally "not recommend any for clinical use". The most frequent bias issues referred to the analysis; however, the most serious ones were those related to sampling. Authors recommend concentrating on avoiding biases in sampling and prioritising the study of already identified predictive factors, rather than the identification of new ones that are often dependent on the database. Our objective is to develop a model to predict which patients with COVID-19 pneumonia are at high risk of developing severe illness or dying, using basic and validated clinical tools.

Page $2 / 30$ 


\section{Methods}

\section{Study design}

Prospective cohort study, formed by all patients consecutively admitted to the Hospital Universitario Virgen de la Victoria (HUVV) with COVID-19 pneumonia, during the first wave: March 1 to April 28, 2020. Follow-up lasted until the discharge of the last patient: July 21,2020. HUVV is a 506-bed hospital, classified as level 2, located in Málaga (southern Spain), which directly serves a population of 470,000 inhabitants.

\section{Participants and source of data}

The inclusion criteria were: confirmed, symptomatic SARS-CoV-2 infection; and requiring hospital admission. Exclusion criteria were: age under 14 years. When the patient had consulted several times at the Emergency Department, data was collected from the consultation in which the acute infection by SARS-CoV-2 was diagnosed.

SARS-CoV-2 infection was confirmed by real-time reverse transcription polymerase chain reaction (RT-PCR), or detection of IgM antibodies with enzyme immunoassay techniques (ELISA).

Hospital admission was based on respiratory symptoms plus radiological infiltrates or significant comorbidity. Admission to Intensive Care Unit (ICU) was based on the development of severe disease and recoverability.

Data were collected from patients or their relatives, the computerised medical record, and the daily handover list of unstable COVID patients in the wards. It was collected within the framework of "International COVID-19 Clinical Evaluation Registry: HOPE-COVID 19" that was evaluated by the Ethics and Research Committee of the Hospital Clínico San Carlos in Madrid. The database records were entered anonymized, with an alphanumeric code and the identifying data were kept in a different file guarded by the local researchers; following data protection laws in force: Ley Orgánica 15/1999, of December 13, de Protección de Datos de Carácter Personal; Ley 41/2002, of November 14, Básica Reguladora de la Autonomía del Paciente y Derechos y Obligaciones en materia de Información y Documentación Clínica. Ley 14/2007, of July 3, de Investigación Biomédica; and Ethical Principles for Medical Research on Human Beings established in the Declaration of Helsinki by the World Medical Association. Written informed consent was waived by the Ethics and Research Committee of the Hospital Clínico San Carlos, due to the nature of the anonymized registry and the severity of the situation.

Model development and reporting followed the TRIPOD (Transparent Reporting of a multivariable prediction model for Individual Prediction Or Diagnosis) guidelines.[2]

\section{Patients and Public involvement}

Patients and public have not been involved in the development of the research question, outcome measures, design nor execution of this study.

\section{Outcomes}

The primary outcome was the development of severe disease, defined by the presence of one of the following criteria: a respiratory failure that needs an inspiratory fraction of oxygen $\left(\mathrm{FiO}_{2}\right)$ equal to or greater than 0.6 , shock or severe dysfunction of another organ, or death. The secondary outcome was vital status at hospital discharge (alive/dead). 


\section{Predictors}

In each patient, we collected demographic characteristics (gender, age, provenance), comorbidities, baseline functional situation and usual medication, the situation at admission (symptoms and signs, complementary examinations), evolution during hospitalisation, and status at discharge.

As an indicator of acute physiological injury, we calculated the CRB scale[3,4] on admission; it is a validated version of the CURB-65 scale;[5] endorsed by British Thoracic Society[6] and NICE,[7,8] (Supplementary Material).

As a summary variable of comorbidity, we calculated the Age-Adjusted Charlson Comorbidity Index[9,10] (AgeCharlson). We chose to evaluate age as a part of the comorbidity index instead of in CRB-65 for two reasons: first, because of clinical significance, as we consider that increasing age provides information about non-explicit comorbidity, it is a kind of "hidden comorbidity index"; and second, for statistical reasons, to minimise the number of predictor variables while maximising the exploitation of a continuous variable (Supplementary Material)

\section{Statistical analysis}

The sample size was determined by the evolution of the pandemic. No imputation of values has been made in the missing data.

In the descriptive analysis, absolute and relative frequencies were calculated in the categorical variables, mean and standard deviation (SD) in the continuous ones with Normal distribution, and median and interquartile range (IQR) in the continuous ones with non-Normal distribution.

For the bivariate analysis, according to the outcomes of interest, we also calculated the relative risks (RR) or odds ratio (OR) and the $95 \%$ confidence intervals.

Multivariate analysis was carried out with forward conditional stepwise logistic regression. Dependent variables were primary or secondary outcomes; independent variables were selected by clinical and statistical criteria in several stages. For statistical analysis, we used the IBM SPSS Statistics package, version 25.

\section{Results}

The first COVID-19 patient was admitted to our hospital on March 1, 2020, and the last of this "first wave" on April 28, 2020; during that period 413 records were included in the database. From them, eight records were deleted because of duplication; twelve patients were excluded because they were transferred to another hospital due to administrative reasons, without being admitted at HUVV; and one patient was excluded because he had an asymptomatic SARS-CoV-2 infection and was hospitalised because of non-related condition (atrioventricular block). Therefore, 392 patients with COVID-19 are analysed. Figure 1 shows the participants flowchart. Follow-up lasted until the discharge of the last patient: July 21,2020 . One hundred and four patients developed severe disease $(27 \%$ of the study group), and fifty-two died (13\%). In the Supplementary Material, Figure S1 shows the daily flow of admissions, discharges and patients hospitalised.

Baseline characteristics are shown in Table 1 and 2. The mean age was 61 years; $59 \%$ were men. The median burden of comorbidity was 2 points in the Age-Charlson scale, being significantly higher in patients who developed severe disease (median 4.5 versus 2 in the non-severe), and in those who died (median 6.5 points versus 2 in those who survived). Fourteen per cent of the patients had some degree of dependency in activities of daily living. The 
most prevalent pathological background was arterial hypertension (46\%). In the bivariate analysis, the variables most clearly associated with the development of severe disease or death were: age (71 years in the severe vs 57 in the non-severe), cerebrovascular disease, chronic heart disease and arterial hypertension (unadjusted RR of developing severe disease between 2.5 and 3, with narrow intervals).

Table 1. Baseline characteristics, of the whole sample and according to the development of severe disease and vital status at discharge. 


\begin{tabular}{|c|c|c|c|c|c|c|c|c|}
\hline \multirow[t]{5}{*}{ Variables } & & \multirow{5}{*}{$\begin{array}{c}\text { Total } \\
\text { n: } \\
392 \\
\text { n } \\
(\%)\end{array}$} & \multicolumn{3}{|c|}{ Disease severity } & \multicolumn{3}{|c|}{ Vital status at } \\
\hline & & & & Severe & & Alive & & \\
\hline & & & n. 288 & n. 104 & & 310 & 57 & \\
\hline & & & & & & & & \\
\hline & & & n (\%) & n (\%) & & $\begin{array}{c}\mathrm{n} \\
(\%)\end{array}$ & n (\%) & \\
\hline \multirow[t]{2}{*}{ Gender } & Women & \multirow{2}{*}{$\begin{array}{l}162 \\
(41) \\
230 \\
(59)\end{array}$} & \multirow{2}{*}{$\begin{array}{c}129 \\
(80) \\
159 \\
(69)\end{array}$} & $33(20)$ & \multirow{2}{*}{$\begin{array}{r}1.5 \\
(1.06- \\
2.17)\end{array}$} & 144 & \multirow{2}{*}{$\begin{array}{r}18 \\
(11) \\
34 \\
(15)\end{array}$} & \multirow{2}{*}{$\begin{array}{r}1.3(0.8- \\
2.3)\end{array}$} \\
\hline & Men & & & $71(31)$ & & $\begin{array}{l}196 \\
(85)\end{array}$ & & \\
\hline Age & $\begin{array}{l}\text { mean } \pm \\
\text { SD }\end{array}$ & $61 \frac{ \pm}{16}$ & $57 \pm \frac{ \pm}{16}$ & $71 \pm 12$ & $\begin{array}{l}1.06^{*} \\
(1.05- \\
1.08)\end{array}$ & $59 \frac{ \pm}{15}$ & $75 \frac{ \pm}{1}$ & $\begin{array}{l}1.09 * \\
\text { (1.06- } \\
1.12)\end{array}$ \\
\hline \multirow[t]{2}{*}{ Chronic lung disease } & No & \multirow{2}{*}{$\begin{array}{r}161 \\
(69) \\
72 \\
(31)\end{array}$} & \multirow{2}{*}{$\begin{array}{r}103 \\
(64) \\
40 \\
(56)\end{array}$} & $58(36)$ & \multirow{2}{*}{$\begin{array}{r}1.2(0.8- \\
1.7)\end{array}$} & 139 & \multirow{2}{*}{$\begin{array}{r}22 \\
(14) \\
24 \\
(33)\end{array}$} & \multirow{2}{*}{$2.4(1.5-$} \\
\hline & Yes & & & $32(44)$ & & $\begin{array}{r}86) \\
48 \\
(67)\end{array}$ & & \\
\hline \multirow[t]{2}{*}{ Chronic heart disease } & No & \multirow{2}{*}{$\begin{array}{r}315 \\
(85) \\
56 \\
(15)\end{array}$} & \multirow{2}{*}{$\begin{array}{r}246 \\
(78) \\
23 \\
(41)\end{array}$} & $69(22)$ & \multirow{2}{*}{$2.6\left(\begin{array}{l}1.9- \\
3.6)\end{array}\right.$} & 284 & \multirow{2}{*}{$\begin{array}{r}31 \\
(10) \\
21 \\
(38)\end{array}$} & \multirow{2}{*}{$\begin{array}{r}3.8 \text { (2.4- } \\
6.1)\end{array}$} \\
\hline & Yes & & & $33(59)$ & & $\begin{array}{r}90) \\
35 \\
(63)\end{array}$ & & \\
\hline \multirow[t]{2}{*}{ Arterial Hypertension } & No & \multirow{2}{*}{$\begin{array}{c}213 \\
(54) \\
179 \\
(46)\end{array}$} & \multirow{2}{*}{$\begin{array}{l}181 \\
(85) \\
107 \\
(60)\end{array}$} & $32(15)$ & \multirow{2}{*}{$2.6\left(\begin{array}{r}1.8- \\
3.8)\end{array}\right.$} & 202 & \multirow{2}{*}{$\begin{array}{r}11 \\
(5) \\
41 \\
(23)\end{array}$} & \multirow{2}{*}{4.4 (2.4- } \\
\hline & Yes & & & $72(40)$ & & $\begin{array}{l}138 \\
138 \\
(77)\end{array}$ & & \\
\hline Obesity & No & 167 & 123 & $44(26)$ & $1.9(1.3-$ & 148 & 19 & $3.1(1.7-$ \\
\hline & Yes & $\begin{array}{r}37 \\
(18)\end{array}$ & $\begin{array}{l}14) \\
18 \\
(49)\end{array}$ & $19(51)$ & & $\begin{array}{r}24 \\
(65)\end{array}$ & $\begin{array}{r}13 \\
135)\end{array}$ & \\
\hline Diabetes mellitus & No & 307 & 246 & $61(20)$ & $2.5(1.8-$ & 274 & 33 & $2.3(1.4-$ \\
\hline & Yes & $\begin{array}{l}025 \\
68 \\
(18)\end{array}$ & 34 & $34(50)$ & & $\begin{array}{r}51 \\
51\end{array}$ & $\begin{array}{r}17 \\
17 \\
(25)\end{array}$ & \\
\hline Chronic kidney disease & No & 369 & 278 & $91(25)$ & $2.2(1.5-$ & 328 & 41 & $4.3(2.6-$ \\
\hline & Yes & $\begin{array}{r}23 \\
(6)\end{array}$ & $\begin{array}{r}10 \\
(43)\end{array}$ & $13(57)$ & & $\begin{array}{r}12 \\
(52)\end{array}$ & $\begin{array}{r}11 \\
(48)\end{array}$ & \\
\hline Cerebrovascular disease & No & 347 & 270 & $77(22)$ & $2.9(2.1-$ & 312 & 35 & $4(2.5-$ \\
\hline & Yes & $\begin{array}{r}89) \\
42 \\
(11)\end{array}$ & $\begin{array}{r}18) \\
15 \\
(36)\end{array}$ & $27(64)$ & & $\begin{array}{r}90) \\
25 \\
(60)\end{array}$ & $\begin{array}{r}17 \\
17 \\
(40)\end{array}$ & \\
\hline Connective tissue disease & No & 375 & 279 & $96(26)$ & $0.9(0.2-$ & 326 & 49 & $1.9(0.6-$ \\
\hline & Yes & $8(2)$ & $6(75)$ & $2(25)$ & & $\begin{aligned}(87) \\
6\end{aligned}$ & $\begin{array}{r}(13) \\
2 \\
(25)\end{array}$ & \\
\hline Chronic liver disease & No & 370 & 274 & $96(26)$ & 1.8 & 324 & 46 & $2.7(1.2-$ \\
\hline & Yes & 15 & $8(53)$ & $7(47)$ & $\begin{array}{r}(1.02- \\
3.1)\end{array}$ & $\begin{array}{l}10 \\
10 \\
(67)\end{array}$ & (12) & \\
\hline Cancer & No & 354 & 262 & $92(26)$ & $1.3(0.8-$ & 310 & 44 & $1.8(0.9-$ \\
\hline & Yes & $\begin{array}{r}91) \\
35 \\
(9)\end{array}$ & $\begin{array}{r}(14) \\
23 \\
(66)\end{array}$ & $12(34)$ & & $\begin{array}{r}88) \\
27 \\
(77)\end{array}$ & $\begin{array}{r}12)^{8} \\
8 \\
(23)\end{array}$ & \\
\hline Immunodepression & No & 359 & 270 & $89(25)$ & $1.4(0.7-$ & 317 & 42 & $3(1.5-$ \\
\hline & Yes & 20 & 13 & 7 (35) & & $\begin{aligned}(00) \\
13 \\
(65)\end{aligned}$ & 7 & \\
\hline $\begin{array}{l}\text { Dependency for Activities } \\
\text { of dailv living }\end{array}$ & No & 330 & 260 & $70(21)$ & $2.4(1.7-$ & 303 & 27 & 5.3 (3.3- \\
\hline & Yes & $\begin{array}{r}53 \\
53 \\
(14)\end{array}$ & $\begin{array}{r}26 \\
(49)\end{array}$ & $27(51)$ & & $\begin{array}{r}30 \\
30\end{array}$ & $\begin{array}{r}23 \\
(43)\end{array}$ & \\
\hline Age-Charlson & $\mathrm{n}$ & 392 & 288 & 104 & $\begin{array}{r}1.4^{*} \\
(1.2-1.5)\end{array}$ & 340 & 52 & $\begin{array}{r}1.6^{*} \\
(1.4-1.8)\end{array}$ \\
\hline
\end{tabular}




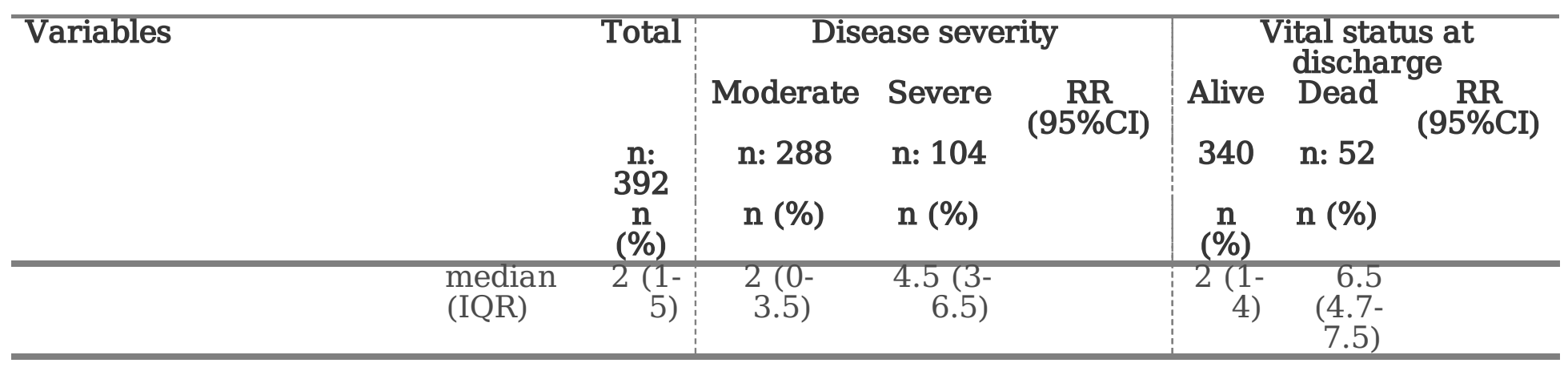

RR: relative risk. IQR: interquartile range. SD: standard deviation. * In the variables Age and AgeCharlson, the association with severe disease or death is reported as OR (95\%CI). In bold and italic those associations that are statistically significant.

Table 2. Home treatment. Descriptive analysis of the whole sample, and depending on the development of severe disease, and the status at discharge.

\begin{tabular}{|c|c|c|c|c|c|c|c|c|}
\hline \multirow{3}{*}{ Variables } & & \multirow{3}{*}{$\begin{array}{l}\text { Total } \\
\text { n: } 392 \\
\text { n (\%) }\end{array}$} & \multicolumn{3}{|c|}{ Disease severity } & \multicolumn{3}{|c|}{ Vital status at discharge } \\
\hline & & & Moderate & Severe & $\mathrm{RR}(95 \% \mathrm{CI})$ & Alive & Dead & RR \\
\hline & & & $\begin{array}{c}\mathrm{n}: 288 \\
\mathrm{n}(\%)\end{array}$ & $\begin{array}{c}\text { n: } 104 \\
\text { n (\%) }\end{array}$ & & $\begin{array}{c}340 \\
\mathrm{n}(\%)\end{array}$ & $\begin{array}{l}\text { n: } 52 \\
\text { n (\%) }\end{array}$ & \\
\hline \multirow[t]{2}{*}{ Antiplatelets } & No & $\begin{array}{l}311 \\
(82)\end{array}$ & $247(79)$ & \multirow{2}{*}{$\begin{array}{r}64 \\
(21) \\
31 \\
(45)\end{array}$} & \multirow[t]{2}{*}{$2.1(1.5-3)$} & \multirow{2}{*}{$\begin{array}{r}284 \\
(91) \\
48 \\
(70)\end{array}$} & $27(9)$ & \multirow{2}{*}{$\begin{array}{r}3.5(2.1- \\
5.8)\end{array}$} \\
\hline & Yes & 69 (18) & $38(55)$ & & & & $\begin{array}{r}21 \\
(30)\end{array}$ & \\
\hline \multirow[t]{2}{*}{ Anticoagulants } & No & \multirow{2}{*}{$\begin{array}{r}355 \\
(93) \\
27(7)\end{array}$} & $273(77)$ & 82 & \multirow[t]{2}{*}{$2.5(1.7-3.7)$} & 315 & 40 & \multirow{2}{*}{$\begin{array}{r}3.6(2.1- \\
6.2)\end{array}$} \\
\hline & Yes & & $11(41)$ & $\begin{array}{l}16 \\
(59)\end{array}$ & & $\begin{array}{r}16 \\
\text { (59) }\end{array}$ & $\begin{array}{r}11 \\
(41)\end{array}$ & \\
\hline \multirow[t]{2}{*}{ Any antithrombotic } & No & 296 & $241(81)$ & 55 & \multirow[t]{2}{*}{$2.6(1.8-3.5)$} & 275 & $21(7)$ & \multirow{2}{*}{$\begin{array}{r}4.8(2.9- \\
7.9)\end{array}$} \\
\hline & Yes & $89(23)$ & $46(52)$ & $\begin{array}{r}43 \\
(48)\end{array}$ & & $\begin{array}{r}59 \\
59 \\
(66)\end{array}$ & $\begin{array}{r}30 \\
(34)\end{array}$ & \\
\hline \multirow[t]{2}{*}{ ARA or ACEi } & No & 243 & $203(84)$ & 40 & \multirow[t]{2}{*}{$2.4(1.7-3.4)$} & $\begin{array}{l}226 \\
226\end{array}$ & $17(7)$ & \multirow[t]{2}{*}{$3.4(2-5.9)$} \\
\hline & Yes & $\begin{array}{l}143 \\
(37)\end{array}$ & $85(59)$ & $\begin{array}{r}58 \\
(41)\end{array}$ & & $\begin{array}{l}109 \\
(76)\end{array}$ & $\begin{array}{r}34 \\
(24)\end{array}$ & \\
\hline \multirow[t]{2}{*}{ BetaBlockers } & No & $\begin{array}{l}336 \\
(87)\end{array}$ & $262(78)$ & 74 & \multirow[t]{2}{*}{$2.2(1.5-3.1)$} & 301 & $\begin{array}{r}35 \\
(10)\end{array}$ & \multirow{2}{*}{3.1 (1.9- } \\
\hline & Yes & 49 (13) & $25(51)$ & $\begin{array}{r}24 \\
(49)\end{array}$ & & $\begin{array}{r}33 \\
(67)\end{array}$ & $\begin{array}{r}16 \\
(33)\end{array}$ & \\
\hline \multirow{2}{*}{$\begin{array}{l}\text { Vitamin D } \\
\text { supplements }\end{array}$} & No & 361 & $273(76)$ & 88 & \multirow[t]{2}{*}{$1.2(0.6-2.4)$} & 317 & 44 & \multirow{2}{*}{$\begin{array}{r}2.1(0.9- \\
4.6)\end{array}$} \\
\hline & Yes & $20(5)$ & $14(70)$ & $6(30)$ & & $\begin{array}{r}15 \\
155)\end{array}$ & $5(25)$ & \\
\hline \multirow[t]{2}{*}{ Benzodiazepines } & No & $\begin{array}{l}313 \\
(81)\end{array}$ & $241(77)$ & $\begin{array}{r}72 \\
(23)\end{array}$ & \multirow[t]{2}{*}{$1.5\left(\begin{array}{c}1.07- \\
2.24)\end{array}\right.$} & $\begin{array}{l}278 \\
(89)\end{array}$ & $\begin{array}{r}35 \\
(11)\end{array}$ & \multirow[t]{2}{*}{$2(1.1-3.3)$} \\
\hline & Yes & 73 (19) & $47(64)$ & $\begin{array}{r}26 \\
(36)\end{array}$ & & $\begin{array}{r}57 \\
(78)\end{array}$ & $\begin{array}{r}16 \\
(22)\end{array}$ & \\
\hline \multirow[t]{2}{*}{ Antidepressant } & No & 323 & $250(77)$ & 73 & \multirow[t]{2}{*}{$1.7(1.2-2.5)$} & 287 & 36 & \multirow{2}{*}{$2.2(1.3-$} \\
\hline & Yes & 62 (16) & $37(60)$ & $\begin{array}{r}25 \\
(40) \\
\end{array}$ & & $\begin{array}{r}47 \\
(76) \\
\end{array}$ & $\begin{array}{l}15 \\
(24) \\
\end{array}$ & \\
\hline
\end{tabular}


ARA: angiotensin II receptor antagonists. ACEi: angiotensin-converting enzyme inhibitor. In bold and italic those associations that are statistically significant.

The situation on arrival at the Emergency Department is summarised in Tables 3 and 4 (Table 3, Table 4). The average duration of symptoms was 7 days (median), being significantly shorter in patients with complications (6 days in those who developed severe disease, and 5 days in those who eventually died). The CRB score was 0 points in more than $80 \%$ of the cases, but any increase was strongly associated with adverse evolution. Baseline pulse oximetry saturation on arrival was the simple complementary examination most strongly associated with a negative outcome in the bivariate analysis.

Table 3. Symptoms and signs at presentation. Total sample and according to the development of severe disease and vital status at discharge. 


\begin{tabular}{|c|c|c|c|c|c|c|c|c|}
\hline \multirow[t]{2}{*}{ Variables } & & \multirow[t]{2}{*}{ Total } & \multicolumn{3}{|c|}{ Disease severity } & \multicolumn{3}{|c|}{ Vital status at } \\
\hline & & & Moderate & Severe & $\begin{array}{c}\mathrm{RR} \\
(95 \% \mathrm{CI})\end{array}$ & Alive & Dead & $\begin{array}{l}\mathrm{RR} \\
(95 \% \mathrm{CI})\end{array}$ \\
\hline \multirow[t]{2}{*}{$\begin{array}{l}\text { Days from symptoms onset } \\
\text { to Hospital admission }\end{array}$} & $\begin{array}{l}\mathrm{n} \\
\text { median } \\
\text { (IQR) }\end{array}$ & $\begin{array}{l}392 \\
7 \text { (4- } \\
10)\end{array}$ & $\begin{array}{l}288 \\
7(4-10)\end{array}$ & $\begin{array}{c}104 \\
6(3- \\
8.2)\end{array}$ & $\begin{array}{c}0.9^{*} \\
(0.8- \\
0.9)\end{array}$ & $\begin{array}{l}340 \\
7(4- \\
10)\end{array}$ & $\begin{array}{c}52 \\
5(3- \\
7.2)\end{array}$ & $\begin{array}{c}0.9^{*} \\
(0.8- \\
0.9)\end{array}$ \\
\hline & & $\begin{array}{c}\mathrm{n} \\
(\%)\end{array}$ & $\mathrm{n}(\%)$ & n (\%) & & n (\%) & $\mathrm{n}(\%)$ & \\
\hline \multirow[t]{2}{*}{ Asymptomatic } & No & $\begin{array}{r}372 \\
(97)\end{array}$ & $275(74)$ & $\begin{array}{r}97 \\
(26)\end{array}$ & $\begin{array}{r}0.3 \\
(0.04-\end{array}$ & $\begin{array}{l}321 \\
(86)\end{array}$ & $\begin{array}{r}51 \\
(14)\end{array}$ & NA \\
\hline & Yes & $\begin{array}{l}13 \\
(3)\end{array}$ & $12(92)$ & $1(8)$ & 1.9) & $\begin{array}{r}13 \\
(100)\end{array}$ & $0(0)$ & \\
\hline \multirow[t]{2}{*}{ Fever } & No & $\begin{array}{r}77 \\
(20)\end{array}$ & $54(70)$ & $\begin{array}{r}23 \\
(30)\end{array}$ & $0.8(0.5-$ & $\begin{array}{r}66 \\
(86)\end{array}$ & $\begin{array}{r}11 \\
(14)\end{array}$ & 0.9 (0.5- \\
\hline & Yes & $\begin{array}{l}304 \\
(80)\end{array}$ & 231 (76) & $\begin{array}{r}73 \\
(24)\end{array}$ & & $\begin{array}{r}265 \\
(87) \\
\end{array}$ & $\begin{array}{r}39 \\
(13)\end{array}$ & \\
\hline \multirow[t]{2}{*}{ Cough } & No & $\begin{array}{r}93 \\
(25)\end{array}$ & $65(70)$ & $\begin{array}{r}28 \\
(30)\end{array}$ & $0.7(0.5-$ & 75 & 18 & $0.5\left(\begin{array}{c}0.3- \\
0.9)\end{array}\right.$ \\
\hline & Yes & $\begin{array}{l}282 \\
(75)\end{array}$ & $216(77)$ & $\begin{array}{r}66 \\
(23)\end{array}$ & & $\begin{array}{l}252 \\
(89)\end{array}$ & $\begin{array}{r}30 \\
(11)\end{array}$ & \\
\hline \multirow[t]{2}{*}{ Dyspnea } & No & 185 & $153(83)$ & 32 & $1.9(1.3-$ & 168 & 17 & $1.9(1.1-$ \\
\hline & Yes & $\begin{array}{l}195 \\
191)\end{array}$ & $129(66)$ & $\begin{array}{r}66 \\
(34)\end{array}$ & & $\begin{array}{l}161 \\
(83)\end{array}$ & $\begin{array}{r}34 \\
(17)\end{array}$ & \\
\hline \multirow[t]{2}{*}{ Tachypnea } & No & 326 & 258 (79) & 68 & $3.2(2.4-$ & 294 & 32 & $3.5(2.1-$ \\
\hline & Yes & $\begin{array}{r}49 \\
(13)\end{array}$ & $16(33)$ & $\begin{array}{r}33 \\
(67)\end{array}$ & & $\begin{array}{r}32 \\
(65)\end{array}$ & $\begin{array}{r}17 \\
(35)\end{array}$ & \\
\hline \multirow[t]{2}{*}{ Asthenia } & No & 152 & $117(77)$ & 35 & $1.2(0.8-$ & 133 & 19 & $1.2(0.7-$ \\
\hline & Yes & $\begin{array}{l}186 \\
(55)\end{array}$ & $132(71)$ & $\begin{array}{r}54 \\
(29)\end{array}$ & & $\begin{array}{l}159 \\
(85)\end{array}$ & $\begin{array}{r}27 \\
(15)\end{array}$ & \\
\hline \multirow[t]{2}{*}{ Myalgias } & No & 160 & $116(73)$ & 44 & 0.8 (0.5- & 140 & 20 & 0.9 (0.5- \\
\hline & Yes & $\begin{array}{l}162 \\
(50)\end{array}$ & $126(78)$ & $\begin{array}{r}36 \\
(22)\end{array}$ & & $\begin{array}{l}143 \\
(88)\end{array}$ & $\begin{array}{r}19 \\
(12)\end{array}$ & \\
\hline \multirow[t]{2}{*}{ Odynophagia } & No & 241 & $175(73)$ & $\begin{array}{r}66 \\
(27)\end{array}$ & 0.2 & 211 & 30 & $0.2(0-$ \\
\hline & Yes & $\begin{array}{r}33 \\
(12)\end{array}$ & $31(94)$ & $2(6)$ & $0.8)$ & $\begin{array}{r}32 \\
(97)\end{array}$ & $1(3)$ & \\
\hline \multirow[t]{2}{*}{ Diarrhoea } & No & 264 & $190(72)$ & $\begin{array}{r}74 \\
(28)\end{array}$ & $0.7\left(\begin{array}{c}0.4- \\
12)\end{array}\right.$ & $\begin{array}{l}225 \\
(85)\end{array}$ & $\begin{array}{r}39 \\
(15)\end{array}$ & $0.5(0.2-$ \\
\hline & Yes & $\begin{array}{r}73 \\
(22)\end{array}$ & $58(79)$ & $\begin{array}{l}15 \\
(21)\end{array}$ & & $\begin{aligned} 68 \\
(93)\end{aligned}$ & $5(7)$ & \\
\hline \multirow[t]{2}{*}{ Anosmia } & No & $\begin{array}{l}198 \\
(94)\end{array}$ & $143(72)$ & $\begin{array}{r}55 \\
(28)\end{array}$ & NA & $\begin{array}{l}166 \\
(84)\end{array}$ & $\begin{array}{r}32 \\
(16)\end{array}$ & NA \\
\hline & Yes & $\begin{array}{l}13 \\
(6)\end{array}$ & $13(100)$ & $0(0)$ & & $\begin{array}{r}13 \\
(100)\end{array}$ & $0(0)$ & \\
\hline \multirow[t]{2}{*}{ Dysgeusia } & No & 195 & $139(71)$ & 56 & 0.2 & 163 & 32 & NA \\
\hline & Yes & $\begin{array}{l}13 \\
(6)\end{array}$ & $12(92)$ & $1(8)$ & 1.7) & $\begin{array}{r}13 \\
1001\end{array}$ & $0(0)$ & \\
\hline \multirow[t]{2}{*}{ Thromboembolism } & No & 365 & $280(77)$ & 85 & $3.6(2.7-$ & 318 & 47 & $2.2(0.9-$ \\
\hline & Yes & $\begin{array}{l}14 \\
(4)\end{array}$ & $2(14)$ & 12 & & 10 & $\begin{array}{r}4 \\
(29)\end{array}$ & \\
\hline \multirow[t]{2}{*}{ Haemoptysis } & No & 376 & $283(75)$ & 93 & NA & 328 & 48 & $5.9(3.1-$ \\
\hline & Yes & $4(1)$ & $0(0)$ & 4 & & & 3 & \\
\hline \multirow[t]{2}{*}{ Heart failure } & No & 356 & $281(79)$ & 75 & $4.1(3.2-$ & 323 & 33 & $8(5.3-$ \\
\hline & Yes & (6) & $3(13)$ & 20 & & $\begin{aligned} 6 \\
(26)\end{aligned}$ & $\begin{aligned} 17 \\
(74)\end{aligned}$ & \\
\hline
\end{tabular}




\begin{tabular}{|c|c|c|c|c|c|c|c|c|}
\hline \multirow[t]{2}{*}{ Variables } & & \multirow[t]{2}{*}{ Total } & \multicolumn{3}{|c|}{ Disease severity } & \multicolumn{3}{|c|}{ Vital status at } \\
\hline & & & Moderate & Severe & $\begin{array}{c}\mathrm{RR} \\
(95 \% \mathrm{CI})\end{array}$ & Alive & Dead & $\begin{array}{l}\mathrm{RR} \\
(95 \% \mathrm{CI})\end{array}$ \\
\hline \multirow[t]{2}{*}{ Baseline $\mathrm{SpO}_{2}<90$} & No & \multirow{2}{*}{$\begin{array}{r}323 \\
(82) \\
69 \\
(18)\end{array}$} & $269(83)$ & 54 & \multirow{2}{*}{$\begin{array}{r}4.3(3.2- \\
5.7)\end{array}$} & \multirow{2}{*}{$\begin{array}{r}302 \\
(93) \\
38 \\
(55)\end{array}$} & \multirow{2}{*}{$\begin{array}{r}21 \\
(7) \\
31 \\
(45)\end{array}$} & \multirow{2}{*}{$\begin{array}{r}6.9(4.2- \\
11.3)\end{array}$} \\
\hline & Yes & & $19(28)$ & $\begin{array}{r}50 \\
(72)\end{array}$ & & & & \\
\hline \multirow[t]{2}{*}{ Glasgow coma score $<15$} & No & 345 & $260(75)$ & 85 & \multirow{2}{*}{$\begin{array}{r}2.9(2.1- \\
4.1)\end{array}$} & \multirow{2}{*}{$\begin{array}{r}310 \\
(90) \\
7 \\
(37)\end{array}$} & \multirow{2}{*}{$\begin{array}{r}35 \\
(10) \\
12 \\
(63)\end{array}$} & \multirow{2}{*}{$\begin{array}{r}6.2(3.9- \\
9.9)\end{array}$} \\
\hline & Yes & $\begin{array}{r}19 \\
15)\end{array}$ & $5(26)$ & $\begin{array}{l}14 \\
(74)\end{array}$ & & & & \\
\hline \multirow[t]{2}{*}{ Arterial Hypotension } & No & 350 & 269 (77) & 81 & \multirow{2}{*}{$\begin{array}{r}2.8(1.8- \\
4.4)\end{array}$} & \multirow{2}{*}{$\begin{array}{r}312 \\
(89) \\
7 \\
(58)\end{array}$} & \multirow{2}{*}{$\begin{array}{r}38 \\
(11) \\
5 \\
(42)\end{array}$} & \multirow{2}{*}{$\begin{array}{r}3.8(1.8- \\
8)\end{array}$} \\
\hline & Yes & $\begin{array}{r}12 \\
(3)\end{array}$ & $4(33)$ & $8(67)$ & & & & \\
\hline \multirow[t]{4}{*}{$\mathrm{CRB}^{* *}$} & 0 & 324 & $264(81)$ & 60 & \multirow{4}{*}{$\begin{array}{c}6.4^{*} \\
(3.7- \\
11.1)\end{array}$} & \multirow{4}{*}{$\begin{array}{r}298 \\
(92) \\
38 \\
(66) \\
4 \\
(50) \\
0(0)\end{array}$} & \multirow{4}{*}{$\begin{array}{r}26 \\
(8) \\
20 \\
(34) \\
4 \\
(50) \\
2 \\
(100)\end{array}$} & \multirow{4}{*}{$\begin{array}{l}4.3^{*} \\
(2.6- \\
7.2)\end{array}$} \\
\hline & 1 & 58 & $23(40)$ & 35 & & & & \\
\hline & 2 & $8(2)$ & $1(13)$ & 7 (88) & & & & \\
\hline & 3 & $2(1)$ & $0(0)$ & $\begin{array}{r}2 \\
(100)\end{array}$ & & & & \\
\hline
\end{tabular}

RR: relative risk. IQR: interquartile range. $\mathrm{SpO}_{2}$ : arterial saturation by pulse oximetry. NA: not applicable. * In these variables, the association with severe illness or death is expressed as OR (95 CI). ** The vast majority of the patients had a score of zero on the CRB scale (83\%), which makes the quartiles and range very uninformative, so for a better description we show the absolute and relative frequencies of all values (0-3); though we analyse it as continuous. In bold and italic those association that are statistically significant.

Table 4. Laboratory and radiology at admission. Descriptive analysis of the sample as a whole, and segmented according to whether they developed severe disease, and status at discharge. 


\begin{tabular}{|c|c|c|c|c|c|c|c|c|}
\hline \multirow[t]{3}{*}{ Variables } & & \multirow{3}{*}{$\begin{array}{c}\text { Total } \\
\text { n: } \\
392\end{array}$} & \multicolumn{3}{|c|}{ Disease severity } & \multicolumn{3}{|c|}{ Vital status at discharge } \\
\hline & & & Moderate & Severe & $\mathbf{R}$ & Alive & Dead & \\
\hline & & & $\mathrm{n}: 288$ & $\mathrm{n}: 104$ & & $\mathrm{n}: 340$ & $\mathrm{n}: 52$ & \\
\hline $\begin{array}{l}\text { Haemoglobin } \\
\mathrm{mmol} / \mathrm{L}\end{array}$ & $\begin{array}{l}\mathrm{n} \\
\text { mean } \pm\end{array}$ & $\begin{array}{r}381 \\
8.7 \pm \\
12\end{array}$ & $\begin{array}{r}285 \\
8.7 \pm 1.2\end{array}$ & $\begin{array}{r}96 \\
8.7 \pm \\
12\end{array}$ & $\begin{array}{r}0.8^{*}(0.6- \\
1.05)\end{array}$ & $\begin{array}{r}330 \\
8.63 \pm \\
1.06\end{array}$ & $\begin{array}{r}51 \\
8.13 \\
+13\end{array}$ & $0.7 *\left(\begin{array}{c}0.5- \\
0.9)\end{array}\right.$ \\
\hline $\begin{array}{l}\text { Leukocytes } \\
\left(\mathrm{x} 10^{6} / \mathrm{L}\right)\end{array}$ & $\begin{array}{l}\mathrm{SD} \\
\text { mean } \pm \\
\mathrm{SD}\end{array}$ & $\begin{array}{r}1.4 \\
382 \\
6.8 \pm \\
4.4\end{array}$ & $\begin{array}{r}285 \\
6.6 \pm 4.5\end{array}$ & $\begin{array}{r}1.2 \\
97 \\
7.3 \pm \\
4.1\end{array}$ & $\begin{array}{r}1.4^{*}(0.8- \\
2.2)\end{array}$ & $\begin{array}{r}1.06 \\
331 \\
6.7 \pm \\
4.4\end{array}$ & $\begin{array}{r} \pm 1.3 \\
51 \\
8 \pm 5\end{array}$ & $\begin{array}{r}1.4^{*}(0.8- \\
2.3)\end{array}$ \\
\hline \multirow{2}{*}{$\begin{array}{l}\text { Lymphocyte } \\
\left(\mathrm{x} 10^{6} / \mathrm{L}\right)\end{array}$} & $\mathrm{n}$ & 380 & 285 & 95 & $0.5 *(0.3-$ & 329 & 51 & $0.3^{*}$ \\
\hline & $\underset{\mathrm{SD}}{\operatorname{mean}} \pm$ & $\begin{aligned} 1.2 \pm \\
0.7\end{aligned}$ & $\begin{array}{l}1.3 \pm \\
0.6)\end{array}$ & $\begin{array}{r}1.07 \pm \pm \\
0.7\end{array}$ & $0.8)$ & $\begin{array}{r}1.3 \pm \\
0.7\end{array}$ & $\begin{array}{r}0.93 \\
\pm 0.6\end{array}$ & $\begin{array}{l}(0.13- \\
0.5)\end{array}$ \\
\hline \multirow[t]{2}{*}{ Platelets $\left(\mathrm{x} 10^{6} / \mathrm{L}\right)$} & $\mathrm{n}$ & 381 & 286 & 95 & $0.97 *(0.9-$ & 332 & 49 & $1 *(0.9-$ \\
\hline & $\underset{\mathrm{SD}}{\operatorname{mean}} \pm$ & $218 \pm$ & $224 \pm 90$ & $202 \pm \frac{ \pm}{94}$ & 1) & $220 \pm$ & $200 \pm$ & 1) \\
\hline \multirow{3}{*}{$\begin{array}{l}\text { Sodium in plasma } \\
(\mathrm{mmol} / \mathrm{L})\end{array}$} & $\mathrm{n}$ & 378 & 282 & 96 & $0.97^{*}$ & 328 & 50 & $1^{*}(0.9-$ \\
\hline & $\underset{\text { SD }}{\text { mean }} \pm$ & $138 \frac{ \pm}{5}$ & $138 \pm 5$ & $138 \frac{t}{6}$ & $\begin{array}{l}(0.93- \\
1.02)\end{array}$ & $138 \frac{t}{5}$ & $138 \frac{ \pm}{7}$ & 1.1) \\
\hline & & $\mathrm{n}(\%)$ & $\mathrm{n}(\%)$ & n (\%) & & n (\%) & $\mathrm{n}(\%)$ & \\
\hline \multirow{2}{*}{$\begin{array}{l}\text { Creatinine }>0.11 \\
\mathrm{mmol} / \mathrm{L}\end{array}$} & No & 305 & $245(80)$ & 60 & $2.5(1.8-$ & 285 & $20(7)$ & $8(4.2-$ \\
\hline & Yes & $\begin{array}{r}75 \\
(20)\end{array}$ & $37(49)$ & $\begin{array}{r}38 \\
(51)\end{array}$ & & $\begin{array}{l}48 \\
(64)\end{array}$ & $\begin{array}{r}27 \\
(36)\end{array}$ & \\
\hline \multirow[t]{2}{*}{$\mathrm{LDH}>4.1 \mathrm{ukat} / \mathrm{L}$} & No & 147 & $129(88)$ & 18 & $2.8(1.7-$ & 140 & $7(5)$ & $3.9(1.8-$ \\
\hline & Yes & $\begin{array}{l}224 \\
(60)\end{array}$ & $147(66)$ & $\begin{array}{r}77 \\
(34)\end{array}$ & & $\begin{array}{l}182 \\
(81)\end{array}$ & $\begin{array}{r}42 \\
(19)\end{array}$ & \\
\hline \multirow[t]{2}{*}{ AST > 0.66 ukat/L } & No & 199 & $158(79)$ & 41 & $1.4(0.9-$ & 181 & $18(9)$ & $1.7(1-$ \\
\hline & Yes & $\begin{array}{l}140 \\
(41)\end{array}$ & $99(71)$ & $\begin{array}{r}41 \\
(29)\end{array}$ & & $\begin{array}{l}118 \\
(84)\end{array}$ & $\begin{array}{r}22 \\
(16)\end{array}$ & \\
\hline \multirow{2}{*}{$\begin{array}{l}\text { Troponin I > } 0.05 \\
\mathrm{mcg} / \mathrm{L}\end{array}$} & No & 71 & $55(77)$ & $\begin{aligned} 16 \\
(23)\end{aligned}$ & $2.5(1.1-$ & $\begin{array}{r}65 \\
692)\end{array}$ & $6(8)$ & $3.4(0.8-$ \\
\hline & Yes & $7(9)$ & $3(43)$ & $4(57)$ & & $5(71)$ & $2(29)$ & \\
\hline \multirow[t]{2}{*}{$\mathrm{CRP}>47.6 \mathrm{nmol} / \mathrm{L}$} & No & 47 & $45(96)$ & $2(4)$ & $6.6(1.7-$ & 46 & $1(2)$ & $70(1-$ \\
\hline & Yes & $\begin{array}{l}(12) \\
335 \\
(88)\end{array}$ & $240(72)$ & $\begin{array}{r}95 \\
(28)\end{array}$ & & $\begin{array}{l}(98) \\
285 \\
(85)\end{array}$ & $\begin{array}{r}50 \\
(15)\end{array}$ & \\
\hline \multirow[t]{2}{*}{ PCT > $500 \mathrm{ng} / \mathrm{L}$} & No & 248 & $189(76)$ & 59 & 3.4 (2.4- & 217 & 31 & $2.5(1.1-$ \\
\hline & Yes & $\begin{array}{r}16 \\
16)\end{array}$ & $3(19)$ & $\begin{array}{l}13 \\
(81)\end{array}$ & & $\begin{array}{r}11 \\
(69)\end{array}$ & $5(31)$ & \\
\hline \multirow[t]{2}{*}{ D-dimer $>0.5 \mathrm{mg} / \mathrm{L}$} & No & 124 & $104(84)$ & 20 & $1.9(1.2-$ & 119 & $5(4)$ & $4.3(1.7-1$ \\
\hline & Yes & $\begin{array}{l}207 \\
(63)\end{array}$ & $142(69)$ & $\begin{array}{r}65 \\
(31)\end{array}$ & & $\begin{array}{l}(96) \\
171 \\
(83)\end{array}$ & $\begin{array}{r}36 \\
(17)\end{array}$ & \\
\hline \multirow{2}{*}{$\begin{array}{l}\text { Ferritin }>0.72 \\
\text { nmol/L }\end{array}$} & No & 85 & $72(85)$ & 13 & $1.4(0.7-$ & 78 & $7(8)$ & $1.1(0.4-$ \\
\hline & Yes & $\begin{array}{l}(61) \\
54 \\
(39)\end{array}$ & $42(78)$ & $\begin{array}{l}(15) \\
12 \\
(22)\end{array}$ & & $\begin{aligned}(92) \\
49 \\
(91)\end{aligned}$ & $5(9)$ & \\
\hline \multirow{2}{*}{$\begin{array}{l}\text { Triglycerides > } 1.69 \\
\mathrm{mmol} / \mathrm{L}\end{array}$} & No & 80 & $64(80)$ & 16 & $1.8(0.8-$ & 73 & 7 (9) & $1.8(0.5-$ \\
\hline & Yes & $\begin{array}{l}(81) \\
19 \\
(19)\end{array}$ & $12(63)$ & $7(37)$ & & $\begin{array}{l}(91) \\
16 \\
(84)\end{array}$ & $3(16)$ & \\
\hline \multirow{2}{*}{$\begin{array}{l}\text { Abnormal chest X- } \\
\text { ray }\end{array}$} & No & 11 & $8(73)$ & $3(27)$ & $0.9(0.3-$ & $9(82)$ & $2(18)$ & $0.7(0.2-$ \\
\hline & Yes & $\begin{array}{l}368 \\
(97)\end{array}$ & 275 (75) & $\begin{array}{r}93 \\
(25)\end{array}$ & & $\begin{array}{r}319 \\
(87)\end{array}$ & $\begin{array}{r}49 \\
(13)\end{array}$ & \\
\hline
\end{tabular}


RR: relative risk. SD: standard deviation. LDH: lactate dehydrogenase. AST: aspartate aminotransferase. CRP: C-reactive protein. PCT: procalcitonin. * In these variables, the association with severe illness or death is expressed as OR (95 CI). In bold and italic those association that are statistically significant.

During hospitalisation, most of the patients received hydroxychloroquine (92\%) and lopinavir/ritonavir (80\%). Drugs aimed at attenuating the inflammatory response (corticosteroids, tocilizumab, interferon) were used less frequently (around 20\%) and preferentially in the most ill patients. Remdesivir was not administered due to availability issues. One hundred and four patients developed severe disease ( $27 \%$ of the sample), at a median of 9 days from the onset of symptoms, forty (10\% of the sample) were admitted to the ICU. Fifty-two (13\%) died, sixteen of them in the ICU (40\% of all admitted to ICU). The median hospital stay of the total sample was 8 days, with two clearly differentiated patterns: shorter stays in patients with moderate disease and in patients who die (median of 7 and 7.5 days respectively), and longer stays in patients who survived despite developing severe disease (median 22 days, IQR: 1342.2); these differences are clinically, epidemiologically, and statistically significant (Figure 2). Table 5 summarises the evolution of data.

Table 5. Evolution during hospitalisation. Descriptive analysis of the total sample and depending on the development of severe disease and status at discharge. 


\begin{tabular}{|c|c|c|c|c|c|c|c|c|}
\hline \multirow[t]{3}{*}{ Variables } & & \multirow{3}{*}{$\begin{array}{l}\text { Total } \\
\text { n: } 392 \\
\text { n (\%) }\end{array}$} & \multicolumn{3}{|c|}{ Disease severity } & \multicolumn{3}{|c|}{$\begin{array}{l}\text { Vital status at } \\
\text { discharge }\end{array}$} \\
\hline & & & \multirow{2}{*}{\multicolumn{2}{|c|}{$\begin{array}{c}\text { Moderate } \\
\text { n: } 288 \\
\text { n (\%) }\end{array}$}} & $\begin{array}{l}\text { Severe } \\
\text { n: } 104\end{array}$ & \multicolumn{3}{|c|}{$\begin{array}{cl}\text { Alive } & \text { Dead } \\
\text { n: } & \text { n: } 52\end{array}$} \\
\hline & & & & & 6) $\underset{(95 \% \mathrm{CD}}{\mathrm{RR}}$ & $\begin{array}{l}\text { n } \\
(\%)\end{array}$ & n (\%) & $\underset{(95 \% \mathrm{CD}}{\mathrm{RR}}$ \\
\hline \multirow[t]{3}{*}{ Corticosteroids } & No & 278 & \multirow{3}{*}{$\begin{array}{r}247 \\
(89) \\
34 \\
(32)\end{array}$} & $31(11)$ & \multirow[t]{3}{*}{$6.1(4.2-8.7)$} & \multirow{3}{*}{$\begin{array}{r}258 \\
(93) \\
75 \\
(70)\end{array}$} & \multirow{3}{*}{$\begin{array}{r}20 \\
(7) \\
32 \\
(30)\end{array}$} & \multirow{3}{*}{$4.2(2.5-$} \\
\hline & Yes & & & & & & & \\
\hline & res & (28) & & $73(68)$ & & & & \\
\hline \multirow[t]{2}{*}{ Hydroxychloroquine } & No & $31(8)$ & \multirow{2}{*}{$\begin{array}{r}21 \\
(68) \\
263 \\
(74)\end{array}$} & $10(32)$ & \multirow[t]{2}{*}{$0.8(0.5-1.4)$} & \multirow{2}{*}{$\begin{array}{r}21 \\
(68) \\
315 \\
(88)\end{array}$} & \multirow{2}{*}{$\begin{array}{r}10 \\
(32) \\
41 \\
(12)\end{array}$} & \multirow{2}{*}{$0.4 \begin{array}{r}(0.2- \\
0.6)\end{array}$} \\
\hline & Yes & $\begin{array}{l}356 \\
(92)\end{array}$ & & $93(26)$ & & & & \\
\hline \multirow[t]{2}{*}{ Lopinavir/ ritonavir } & No & 79 & \multirow{2}{*}{$\begin{array}{r}61 \\
(77) \\
223 \\
(72)\end{array}$} & $18(23)$ & \multirow[t]{2}{*}{$1.2(0.8-1.9)$} & \multirow{2}{*}{$\begin{array}{c}65 \\
(82) \\
271 \\
(88)\end{array}$} & \multirow{2}{*}{$\begin{array}{r}14 \\
(18) \\
37 \\
\end{array}$} & \multirow{2}{*}{ 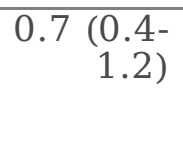 } \\
\hline & Yes & $\begin{array}{l}(20) \\
308 \\
(80)\end{array}$ & & $85(28)$ & & & & \\
\hline \multirow[t]{2}{*}{ Interferon } & No & 318 & \multirow{2}{*}{$\begin{array}{r}259 \\
(81) \\
24 \\
(35)\end{array}$} & $59(19)$ & $3.5(2.6-4.7)$ & 281 & 37 & 1.8 \\
\hline & Yes & \begin{tabular}{r|r}
$(82)$ & \\
68 &
\end{tabular} & & $44(65)$ & & $\begin{array}{r}88) \\
54 \\
(79)\end{array}$ & $\begin{array}{l}(12) \\
14 \\
(21)\end{array}$ & $\begin{array}{l}(.01- \\
3.1)\end{array}$ \\
\hline Tocilizumab & No & $\begin{array}{l}339 \\
(899\end{array}$ & 281 & $58(17)$ & NA & 304 & 35 & $3.5(2.1-$ \\
\hline & Yes & $\begin{array}{r}44 \\
(11)\end{array}$ & $0(0)$ & $44(100)$ & & $\begin{array}{r}28 \\
(64)\end{array}$ & $\begin{array}{r}16 \\
(36)\end{array}$ & \\
\hline $\begin{array}{l}\text { High flow nasal } \\
\text { nronos }\end{array}$ & No & 367 & 283 & $84(23)$ & NA & 326 & 41 & $4.5(2.6-$ \\
\hline & Yes & $18(5)$ & $0(0)$ & $18(100)$ & & $\begin{array}{r}9 \\
(50)\end{array}$ & $\begin{array}{r}9 \\
(50)\end{array}$ & \\
\hline Non-invasive & No & $\begin{array}{l}367 \\
05\end{array}$ & 276 & $91(25)$ & $\mathrm{NA}$ & 320 & 47 & $2(0.9-$ \\
\hline & Yes & $20(5)$ & $\begin{array}{r}7 \\
7 \\
(35)\end{array}$ & $13(65)$ & & $\begin{array}{r}(8 /) \\
15 \\
(75)\end{array}$ & $\begin{array}{l}(13) \\
5 \\
(25)\end{array}$ & \\
\hline Invasive ventilation & No & 350 & 282 & $68(19)$ & NA & 314 & 36 & $4.3(2.7-$ \\
\hline & Yes & $36(9)$ & $0(0)$ & $36(100)$ & & 20 & 16 & \\
\hline ICU admission & No & 352 & NA & NA & NA & 316 & 36 & $3.9(2.4-$ \\
\hline & Yes & $\begin{array}{r:r}40 \\
(10)\end{array}$ & $\begin{array}{r}58 \\
(79)\end{array}$ & $15(21)$ & & $\begin{array}{r}24 \\
(60)\end{array}$ & $\begin{array}{r}16 \\
(40)\end{array}$ & \\
\hline Days symptoms onset & $\mathrm{n}$ & 40 & NA & NA & $\overline{\mathrm{NA}}$ & 24 & 16 & $1 *(0.8-$ \\
\hline to İC & $\begin{array}{l}\text { median } \\
\text { (IQR) }\end{array}$ & \begin{tabular}{r:|r}
$9(6-$ & \\
$12)$ &
\end{tabular} & $\begin{array}{r}13 \\
(100)\end{array}$ & $0(0)$ & & $\begin{array}{r}9 * \\
(6.7- \\
12)\end{array}$ & $\begin{array}{r}9 \\
(5.7- \\
11.2)\end{array}$ & 1.2) \\
\hline APACHE-II at ICU & . & 40 & NA & NA & NA & 24 & 16 & $1.2^{*}$ \\
\hline admi & $\begin{array}{l}\operatorname{mean} \\
\text { (SD) }\end{array}$ & \begin{tabular}{l|l}
11.3 & \\
$(4.7)$ &
\end{tabular} & $\begin{array}{r}12 \\
(92)\end{array}$ & $1(8)$ & & $\begin{array}{l}10 \\
(5)\end{array}$ & $\begin{array}{l}13 \\
(4)\end{array}$ & $(0,9-1.3)$ \\
\hline Length of stay in ICU & $\mathrm{n}$ & 40 & & & NA & 24 & 16 & $0.99 *$ \\
\hline (day & $\begin{array}{l}\text { median } \\
\text { (IQR) }\end{array}$ & $\begin{array}{r:}17.5 \\
(13- \\
35)\end{array}$ & NA & NA & & $\begin{array}{r}26 \\
(13- \\
40)\end{array}$ & $\begin{array}{l}16.5 \\
(12.5- \\
23.7)\end{array}$ & $\begin{array}{l}(0.95- \\
1.01)\end{array}$ \\
\hline Mortality in ICU & & $\begin{array}{r:r}16 / & \\
40 & \\
(40) & \end{array}$ & NA & NA & NA & $\begin{array}{r}24 \\
(60)\end{array}$ & $\begin{array}{r}16 \\
(40)\end{array}$ & NA \\
\hline Leng & $\mathrm{n}$ & 392 & 288 & 104 & $1.14 *(1.1-$ & 340 & 52 & $1.01^{*}$ \\
\hline hoss & $\begin{array}{l}\text { median } \\
\text { (IQR) }\end{array}$ & $\begin{array}{r:}8(5-1 \\
12.25)\end{array}$ & $\begin{array}{l}7(5- \\
10)\end{array}$ & $13(7-26)$ & 1.18) & $\begin{array}{r}8(5- \\
12)\end{array}$ & $\begin{array}{r}7.5 \\
(4- \\
18.2)\end{array}$ & $\begin{array}{l}(0.99- \\
1.02)\end{array}$ \\
\hline $\begin{array}{l}\text { Length of stay in } \\
\text { hospital* for severe } \\
\text { disease only (days) }\end{array}$ & $\mathrm{n}$ & 104 & NA & NA & NA & 52 & 52 & $\begin{array}{l}0.94^{*} \\
(0.91- \\
0.97)\end{array}$ \\
\hline
\end{tabular}




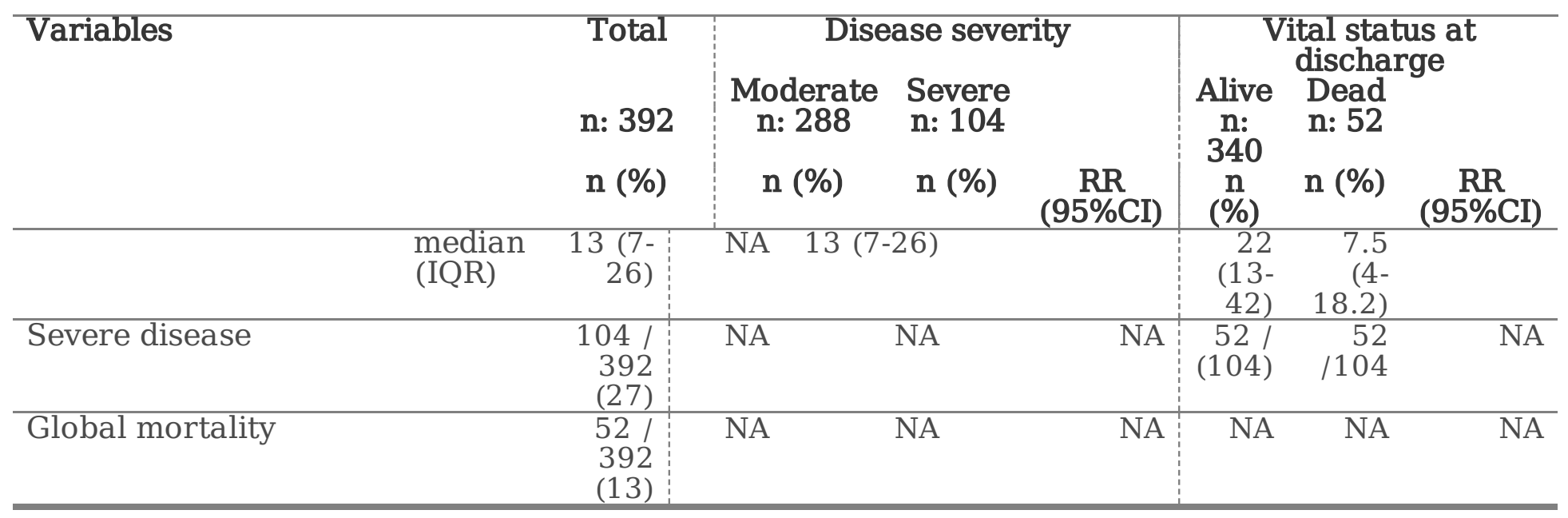

RR: relative risk. SD: standard deviation. IQR: interquartile range. NA: not applicable * In these variables, the association with severe illness or death is expressed as OR (95 CI). In bold and italic those association that are statistically significant.

The final multivariate model for prediction of the primary outcome (development of severe disease), is shown in Table 6. It contains only three variables: Age-Charlson scale, CRB scale, and baseline desaturation by pulse oximetry. There are no missing data, so 392 patients are analysed. Cox and Snell's $\mathrm{R}^{2}$ is 0.28 , and Nagelkerke's 0.42 ; HosmerLemeshow test $p=0.22$; $C$ statistic: 0.85 (95\% Cl: 0.80-0.89), global sensitivity: $93 \%$, specificity: $55 \%$. Figure 3 displays the receiver operating characteristic curve (ROC curve). Logistic regression requirements are met. Among the rest of the factors that could have an independent prognostic value, only CRP, LDH and lymphopenia had statistically significant coefficients but did not improve the overall performance of the model (Supplementary Material, Table S1 and Figure S2). Gender, hypertension, previous dependence, days from onset of symptoms to arrival at the hospital, DD, AST, or acute renal failure upon admission were not significant; troponin and ferritin cannot be evaluated in the multivariate analysis because there are few cases with valid data in the first day. We also do not evaluate drug therapy because their administration has been highly biased by the severity perceived by the physician assisting the patient, and it was not possible to control this confounding factor.

Table 6. Multivariate model for predicting the development of severe disease.

\begin{tabular}{lrrrrr}
\hline & B & Sig. & OR & \multicolumn{2}{c}{ 95\%CI for OR } \\
\cline { 5 - 6 } & & & & Lower & Upper \\
\hline Age-Charlson & 0.253 & $<0.001$ & 1.28 & 1.16 & 1.42 \\
\hline $\mathrm{CRB}$ & 1.427 & $<0.001$ & 4.16 & 2.26 & 7.65 \\
\hline Baseline $\mathrm{SpO}_{2}<90$ & 1.866 & $<0.001$ & 6.46 & 3.32 & 12.53 \\
\hline Constant & -2.676 & $<0.001$ & 0.069 & & \\
\hline
\end{tabular}

In the multivariate analysis for prediction of the secondary outcome (death), we arrived at a model with the same predictors, and remarkably similar performance, Table 7. Figure 4. Hosmer Lemeshow test $p=0.85$; Cox and Snell's $R^{2}: 0.24$ and Nagelkerke's 0.45 . The $C$ statistic: 0.90 (95\% Cl: $0.86-0.94$ ), overall sensitivity $97 \%$, specificity $40 \%$. Among the rest of the variables that could be independent risk factors, only LDH and lymphocyte count reached statistical significance but did not significantly improve the model (Supplementary Material, Table S2 and Figure S3). Gender, arterial hypertension, dependence in activities of daily living, DD, CRP, PCT, AST, leukocytes, haemoglobin, platelets, sodium, acute renal failure or days from the onset of symptoms to arrival at the hospital 
were not significant; troponin or ferritin cannot be explored in the multivariate model because there are few cases with valid data.

Table 7. Multivariate model for predicting death.

\begin{tabular}{lrrrrr}
\hline & B & Sig. & OR & \multicolumn{2}{c}{ 95\%CI for OR } \\
\cline { 4 - 6 } & & & & Lower & Upper \\
\hline Age-Charlson & 0.424 & $<0.001$ & 1.52 & 1.32 & 1.76 \\
\hline CRB & 1.091 & 0.001 & 2.97 & 1.55 & 5.69 \\
\hline Baseline $\mathrm{SpO}_{2}<90$ & 1.636 & $<0.001$ & 5.13 & 2.44 & 10.77 \\
\hline Constant & -4.609 & $<0.001$ & 0.01 & & \\
\hline
\end{tabular}

\section{Discussion}

The main conclusion of this study is that the prognosis of a patient with COVID-19 pneumonia can probably be predicted early by combining a widely validated comorbidity scale and an acute disease scale; the only "complementary examination" that we include is arterial saturation by pulse oximetry, a measurement that can be done at patient's home as easily as taking blood pressure. We have chosen the most popular comorbidity scale: the Charlson Comorbidity Index[11] (age-adjusted version[9]); and as a pneumonia severity scale, one of the CURB-65 family: the CRB scale;[3] but surely there will be other options. The main point is to check the validity of an idea with such clinical coherence: the prognosis of a patient essentially depends on the balance between the resistance capacity and the aggressiveness of the acute problem.

The sample we study meets the requirements to be considered representative: confirmed cases, consecutively included, in the same phase of the disease (on admission to hospital), with homogeneous admission criteria, in a naturally delimited time frame, with prospective data collection and complete follow-up (minimal percentage of losses: 12/404, 3\%). Furthermore, looking at the proportion of hospital beds occupied by COVID-19 patients (maximum 38\%, Supplementary Material, Figure S1), we get the impression that the confounding effect that work overload could have on patient outcomes has been lower in our hospital than in other cases.[12,13]

Baseline characteristics also support the idea of representativeness; they are very similar to those of other series, $[12,14,15]$ predominantly male, with a mean age of 60 years, similar to the USA[16] and intermediate between that of China[17] (around 55 years), and United Kingdom[18] (70 years old). The comorbidity burden was low (Age-Charlson median 2 points), similar to that observed by Casas-Rojo[15] in Spain, and in other series that have evaluated age and the Charlson index separately: Italy,[19] USA,[20] Denmark,[21] or China.[13] In all of them, with such different socio-geographic contexts, both characteristics were independent risk factors, which reinforces the idea of the suitability of combining them in Age-Charlson.

In the first clinical evaluation, $\mathrm{CRB}$ and $\mathrm{SpO}_{2}$ were abnormal in only $18 \%$ of the patients, but with a strong association with severity. $\mathrm{SpO}_{2}$ could be especially useful in COVID-19 patients, helping to detect what has been called "silent hypoxemia".[22,23]

The most widespread model in which data on comorbidity and acute disease are combined in patients with pneumonia is the PSI scale.[24] However, it has a substantial disadvantage: it cannot be used outside a health centre since 7 of its 19 variables require laboratory or radiology/ultrasound. There are very few studies with predictive models applicable in primary care that, at the same time, implement such intuitive idea as that assessing the prognosis of potentially seriously ill patients requires considering not only the aggressiveness of the acute disease but also the burden of chronic disease that weakens them.[25] Generally, both components have been 
studied as alternatives,[26] and rarely as complementary.[27,28] In patients with COVID-19, Petrilli[29] and ISARIC[18,30] are two groups that more closely resemble this study's objective. Petrilli does not explicitly include a comorbidity scale but empirically reaches the same conclusions: age, comorbidity, oxygenation and inflammation parameters determine the need for hospitalisation and the development of severe disease; the relative weight of each possibly varies depending on the outcome and the population of interest. ISARIC-4C is based on the components of the Charlson Index and CURB-65, along with gender, obesity and CRP to build a model with 8 predictor variables, including 2 biochemical which limits its application outside the hospital context; unexpectedly, hypotension has not reached the final model. In polypathological COVID-19 patients, the usefulness of combining acute damage and comorbidity scales has also been partially reported, in this case not with the Charlson index but with a specific scale for polypathological patients (PROFUND).[31]

Regarding other variables that could be important, we have explored the baseline functional situation in terms of dependency for activities of daily living, and though it was significant in the univariate and bivariate analysis (Table 1), it ceased to be so in the multivariate after incorporating the Age-Charlson scale; however, we think it deserves to be further explored. Casas-Rojo[15] have terribly similar results: $16 \%$ of dependency for activities of daily living, and association with worse evolution in the bivariate analysis; the multivariate analysis has yet to be published. Bernabeu-Wittel[31] in a study focused on multiple pathological patients with COVID-19 incorporates functional status (Barthel index) into the assessment of comorbidity.

The rate of severe disease in this series is $27 \%$; in other studies, it ranges between $15 \%$ and $37 \%$. $[29,30,32,33]$ This variability may be due to differences in the selected sample, in the definition of severe disease or in the method used to build the model:

- Major differences in sampling: due to differences in the age of the patients (which we will address next); or due to exclusively including patients diagnosed by chest CT[34] (which is more sensitive than plain radiography); or excluding patients who already present in a severe condition[35-38] (because their objective is to study the progression from non-severe to severe); or limiting follow-up to a short period which does not allow to reach the outcome of interest to a significant proportion of included patients, $[39,40]$ and therefore rising significant risk of selection bias that will be later discussed.

- Important differences in the definition of severe disease: most of the predictive models developed in China $[37,40,41]$ use the definition recommended by the National Health Commission of China, that is broader than ours. In this Chinese definition, a ratio of arterial oxygen pressure to inspiratory oxygen fraction (Pa/Fi) less than 300 is a sufficient criterion to diagnose severe pneumonia. So, for example, a patient that with a FiO2 of 0.3 had a $\mathrm{PaO} 2$ of $80 \mathrm{mmHg}(\mathrm{Pa} / \mathrm{Fi}: 80 / 0.3=267)$, should be considered severe with the Chinese definition, but not with ours. Our definition adopts criteria routinely recommended considering the admission of a patient with pneumonia to an area of high dependency or an Intensive Care Unit,[42-44] regarding $\mathrm{FiO}_{2}$ it requires to need 0.6 or more. Other studies limit the definition to admission in ICU or Intermediate Unit;[18] overlooking that in order to admit a patient in these units, in addition to severity, patient recoverability and availability of beds are also assessed; this explains the variability in the use of ICUs and why a high percentage of severely ill patients are not treated in ICU,[45] approximately $60 \%$ in our series.

- Important differences in the strategy to build prediction models: those studies whose model is based on tools with little availability today, such as artificial intelligence or computer applications with copyright.[34,36,37,46,47] 
Crude mortality rate in our series is $13 \%$ (of hospitalised patients). Again, direct comparison with other series is difficult, even being mortality a more robust outcome than disease severity. In Spain, mortality in multicentre studies of hospitalised patients has been 21-28\%,[15,32] in the United Kingdom 30\%,[30] Italy 20\%.[48] Age distribution and incomplete follow-up are two factors that could explain not only differences in raw mortality but also in the performance of predictive models.

Mortality varies according to age in all series; in our study, it ranges from $0 \%$ under 40 years to almost $40 \%$ at ages above 80 years, Figure 5. A partial solution to improving comparability could be the age-standardised mortality rate, that is the mortality that a population would have if it had the age distribution of a reference population (e.g., the WHO World Standard Population),[49,50] although it is not without criticism.[51] In this series, the age-standardised mortality rate with this reference population is 2.9 deaths per 100 COVID-19 patients admitted.

Incomplete follow-up cannot be controlled in the analysis phase. In mortality studies published in the first months of the pandemic, it has been frequent for the follow-up to be limited to 2 weeks of hospital stay; so that only those patients who have died or been discharged during that time were analysed and those who remained hospitalised were excluded.[16,18,52] The lack of follow-up information on these, most likely biases the estimation of crude mortality and the estimation of the performance of predictive models of mortality.[1,33] Figure 2 shows the distribution of hospital stay in our series depending on whether the patient had moderate disease, severe but finally survived, or severe and finally died; as previously mentioned, the group of severe but surviving patients had the longest stay, well above 14 days, so would be largely censored for the analysis if the follow-up was limited to two weeks. These patients constitute an "informative right censoring" because they are a "selective" loss of survivors, which leads us to calculate an inaccurate higher mortality. And they are also a "selective" loss of patients with a difficult prognosis (they were severely ill but survived), which would lead to prediction models that work better in the study than in real life, since in the study mainly remain patients from the extremes of the spectrum of severity: survivors after mild-moderate illness (left section of the graph), and deceased (right section of the graph). Our series only has a $3 \%$ loss of included patients, and not related to the length of stay nor outcome, but due to transfer from the Emergency Department to another hospital because of their place of residence.

Concerning laboratory variables, we observed that high levels of CRP, PCT and LDH, or

lymphopenia reach statistical significance but do not clinical significance from our perspective. This does not mean that would not be efficient in studies with different objectives; probably comorbidity variables be more decisive in countries with an older population; while variables of acute inflammatory damage do so in countries with a young population[29] This is something that should be investigated, however, is out of scope for this study.

What use can these models have? An essential requirement to apply them with confidence is their validation in independent but representative samples. Once validated, it can have multiple applications, both in the clinical and management area:

- Support to make clinical decisions when, after a routine initial assessment, the course of action is unclear. The predicted probability of severe disease can help to decide whether to admit in the hospital and in which department; or which specific treatment to prescribe.

- Support in decision-making for the management of the infrastructure necessary for the assistance to function as efficiently and effectively as possible.

- Quality control, through the relationship between observed and expected mortality according to the model.[53] 


\section{Study limitations.}

We have studied a sample from a single centre. The sample size is modest, and even though it is sufficient for the study's objective, it still gives us wide confidence intervals in relevant variables. It is possible that some severely ill patients be misclassified as non-severe, specifically those severe ill patients that survived being treated outside the ICU if they were not discussed in the COVID commission. Anyway, if it exists, it will constitute a case of differential misclassification of the outcome, where the expected effect would be to bias the estimates towards null, and therefore it would not weaken the study's conclusions. It is necessary to validate the proposed model, especially with patients evaluated out of the hospital and subsequently followed up until the end of the disease.

\section{Conclusion}

In patients with COVID-19 pneumonia, the prognosis can likely be significantly narrowed by combining a comorbidity scale and a current severity scale of pneumonia, both widely validated. This study proposes a predictive model based on Age-Adjusted Charlson index, the CRB scale, and baseline arterial saturation. It can be completed at the first medical contact through standard anamnesis, physical examination, and a pocket pulse oximeter.

\section{List Of Abbreviations}

95\% Cl: 95\% Confidence Interval.

Age-Charlson: Age-adjusted Charlson Comorbidity Index.

AST: ASpartate Transaminase.

COVID-19: Coronavirus disease 2019.

CRB score: Confussion, Respiratory rate and Blood pressure score.

CRB-65: Confussion, Respiratory rate Blood pressure and Age score.

CRP: C-Reactive Protein.

CT: Computed Tomography.

CURB score: Confussion, Urea, Respiratory rate and Blood pressure score.

CURB-65: Confussion, Urea, Respiratory rate Blood pressure and Age score.

DD: D-Dimer.

ELISA: Enzyme immunoassay technique.

$\mathrm{FiO}_{2}$ : Fraction of Inspired Oxygen.

HOPE-COVID 19: Health Outcome Prediction Evaluation for COVID-19.

HUVV: Hospital Universitario Virgen de la Victoria.

ICU: Intensive Care Unit. 
IQR: InterQuartile Range.

ISARIC: International Severe Acute Respiratory and Emerging Infection Consortium.

NICE: National Institute for Health and Care Excellence.

LDH: Lactate DesHydrogenase.

OR: Odds Ratio.

$\mathrm{Pa} / \mathrm{Fi}$ : ratio of arterial oxygen pressure to inspiratory oxygen fraction.

PCT: ProCalciTonin.

ROC curve: Receiver Operating Characteristics curve.

RR: Relative Risk.

RT-PCR: Real Time Reverse Polymerase Chain Reaction.

SARS-CoV-2: Severe Acute Respiratory S yndrome CoronaVirus 2.

SD: Standard Deviation.

$\mathrm{SaO}_{2}$ : Arterial Oxygen Saturation.

$\mathrm{SpO}_{2}$ : Peripheral capillary Oxygen Saturation.

TRIPOD: Transparent Reporting of multivariable prediction model for Individual Prediction Or Diagnosis.

WHO: World Health Organization.

\section{Declarations}

\section{ETHICS APPROVAL AND CONSENT TO PARTICIPATE}

This study has been done within the framework of "International COVID-19 Clinical Evaluation Registry: HOPE-COVID 19" project that was approved by the Ethics and Research Committee of Hospital Clínico San Carlos in Madrid (20/241-E) and Agencia Española de Medicamentos y productos Sanitarios (EPA-=D). The database records were entered anonymized, with an alphanumeric code and the identifying data were kept in a different file guarded by the local researchers; following data protection laws in force: Ley Orgánica 15/1999, of December 13, de Protección de Datos de Carácter Personal; Ley 41/2002, of November 14, Básica Reguladora de la Autonomía del Paciente y Derechos y Obligaciones en materia de Información y Documentación Clínica. Ley 14/2007, of July 3, de Investigación Biomédica; and Ethical Principles for Medical Research on Human Beings established in the Declaration of Helsinki by the World Medical Association. Written informed consent was waived because the characteristics of the anonymized registry and the severity of the situation.

\section{CONSENT OF PUBLICATION}

Non applicable. 
AVAILABILITY OF DATA AND MATERIALS

The datasets supporting the conclusions of this article are includede within the article and its additional file. Futher details are available from the corresponding author, PNO, upon reasonable request.

\section{COMPETING INTERESTS, FUNDING}

All authors have completed the ICMJE uniform disclosure form at www.icmje.org/coi_disclosure.pdf and declare: no support from any organisation for the submitted work; no financial relationships with any organisations that might have an interest in the submitted work in the previous three years; no other relationships or activities that could appear to have influenced the submitted work.

\section{Authors Contributions}

PNO: conceptualization, design, coordination, result interpretation, draft writing and critical review. CRA: patient care and data acquisition. FDM: patient care and data acquisition. VMBM: design and coordination. LRF: analysis and result interpretation, MAEF: conceptualization.

\section{ACKNOWLEDGMENTS}

We want to state our acknowledgement to all the hospital staff, who have made a special effort to assist these patients. Last but not least, we want to dedicate a mention to all patients and their families; they entrusted us with what they loved most.

\section{COLLABORATORS:}

- Murcia-Casas, B. Collected data and provided and cared for study patients.

- Martinez-Mesa, A. Collected data and provided and cared for study patients.

- Cabrera-Cesar, E. Collected data and provided and cared for study patients.

- Gomez-Perez, AM. Collected data and provided and cared for study patients.

- Aguilar-Galvez, AM. Collected data and provided and cared for study patients.

- Doncel-Abad, V. Collected data and provided and cared for study patients.

- Rodriguez-Capitan, J. Collected data and provided and cared for study patients.

- Vera-Sanchez, MC. Collected data and provided and cared for study patients.

- Gonzalez-Redondo, P. Collected data and provided and cared for study patients.

- Sanchez-Alvarez, E. Collected data and provided and cared for study patients.

- Puerto-Morlan, A. Provided and cared for study patients.

- Zamboschi, NA. Provided and cared for study patients.

- De La Torre-Muñoz, A. Provided and cared for study patients.

- Nieto-Gonzalez, M. Provided and cared for study patients.

- Moratalla-Cecilia, G. Provided and cared for study patients.

- Segura-Gonzalez, F. Provided and cared for study patients.

- Martinez-Lopez, P. Provided and cared for study patients.

- Salido-Diaz, L. Provided and cared for study patients. 
- Cordon-Alvarez, S. Provided and cared for study patients.

- Daga-Ruiz, D. Provided and cared for study patients.

- Salazar-Ramirez, C. Provided and cared for study patients.

- Rueda-Molina, C. Provided and cared for study patients.

- Mateos-Rodriguez, M. Provided and cared for study patients.

- Sanchez-Calderon, A. Provided and cared for study patients.

- Sanchez-Garcia, A. Provided and cared for study patients.

- Fernandez-Villalba, A. Provided and cared for study patients.

- Garcia-Gomez, IG. Provided and cared for study patients.

- Cota-Delgado, C. Provided and cared for study patients.

- Vallejo-Baez, A. Provided and cared for study patients.

- Castillo-Caballero, JM. Provided and cared for study patients.

- Valera-Rubio, M. Provided and cared for study patients.

- Lara-Dominguez, P. Provided and cared for study patients.

- Estevez-Escobar, E. Provided and cared for study patients.

- Garcia-Aragon, S. Provided and cared for study patients.

- Perez-Lopez, C. Provided and cared for study patients.

- Barrera-Serrano, R. Provided and cared for study patients.

- Guerrero-Orriach, JL Provided and cared for study patients.

\section{References}

1. Wynants L, Van Calster B, Collins GS, Riley RD, Heinze G, Schuit E, et al. Prediction models for diagnosis and prognosis of covid-19 infection: systematic review and critical appraisal. BMJ. 2020;369: m1328. doi:10.1136/bmj.m1328

2. Collins GS, Reitsma JB, Altman DG, Moons KGM. Transparent Reporting of a multivariable prediction model for Individual Prognosis Or Diagnosis (TRIPOD): the TRIPOD Statement. Br J Surg. 2015;102: 148-158. doi:10.1002/bjs.9736

3. Bauer TT, Ewig S, Marre R, Suttorp N. CRB-65 predicts death from community-acquired pneumonia. J Intern Med. 2006. Available: https://onlinelibrary.wiley.com/doi/abs/10.1111/j.1365-2796.2006.01657.x

4. Fan G, Tu C, Zhou F, Liu Z, Wang Y, Song B, et al. Comparison of severity scores for COVID-19 patients with pneumonia: a retrospective study. Eur Respir J. 2020. doi:10.1183/13993003.02113-2020

5. Lim WS, van der Eerden MM, Laing R, Boersma WG, Karalus N, Town GI, et al. Defining community acquired pneumonia severity on presentation to hospital: an international derivation and validation study. Thorax. 2003;58: 377-382. doi:10.1136/thorax.58.5.377

6. Lim WS, Baudouin SV, George RC, Hill AT, Jamieson C, Le Jeune I, et al. BTS guidelines for the management of community acquired pneumonia in adults: update 2009. Thorax. 2009;64 Suppl 3: iii1-55. doi:10.1136/thx.2009.121434

7. National Institute for Health and Care Excellence (UK). Pneumonia in adults: diagnosis and management. London: National Institute for Health and Care Excellence (UK); 2020. Available: 
https://www.ncbi.nlm.nih.gov/pubmed/31940163

8. Overview | COVID-19 rapid guideline: managing suspected or confirmed pneumonia in adults in the community | Guidance | NICE. [cited 30 Aug 2020]. Available: https://www.nice.org.uk/guidance/ng165

9. Charlson M, Szatrowski TP, Peterson J, Gold J. Validation of a combined comorbidity index. J Clin Epidemiol. 1994;47: 1245-1251. doi:10.1016/0895-4356(94)90129-5

10. de Groot V, Beckerman H, Lankhorst GJ, Bouter LM. How to measure comorbidity. a critical review of available methods. J Clin Epidemiol. 2003;56: 221-229. doi:10.1016/s0895-4356(02)00585-1

11. Charlson ME, Pompei P, Ales KL, MacKenzie CR. A new method of classifying prognostic comorbidity in longitudinal studies: development and validation. J Chronic Dis. 1987;40: 373-383. doi:10.1016/00219681(87)90171-8

12. Borobia AM, Carcas AJ, Arnalich F, Álvarez-Sala R, Monserrat-Villatoro J, Quintana M, et al. A Cohort of Patients with COVID-19 in a Major Teaching Hospital in Europe. J Clin Med Res. 2020;9. doi:10.3390/jcm9061733

13. Liang W, Liang H, Ou L, Chen B, Chen A, Li C, et al. Development and Validation of a Clinical Risk Score to Predict the Occurrence of Critical IIIness in Hospitalized Patients With COVID-19. JAMA Intern Med. 2020. doi:10.1001/jamainternmed.2020.2033

14. Martos Pérez F, Luque del Pino J, Jiménez García N, Mora Ruiz E, Asencio Méndez C, García Jiménez JM, et al. Comorbilidad y factores pronósticos al ingreso en una cohorte COVID-19 de un hospital general. Rev Ordem Med. 2020. doi:10.1016/j.rce.2020.05.017

15. Casas-Rojo JM, Antón-Santos JM, Millán-Núñez-Cortés J, Lumbreras-Bermejo C, Ramos-Rincón JM, Roy-Vallejo E, et al. Características clínicas de los pacientes hospitalizados con COVID-19 en España: resultados del Registro SEMI-COVID-19. Rev Clin Esp. 2020. doi:10.1016/j.rce.2020.07.003

16. Richardson S, Hirsch JS, Narasimhan M, Crawford JM, McGinn T, Davidson KW, et al. Presenting Characteristics, Comorbidities, and Outcomes Among 5700 Patients Hospitalized With COVID-19 in the New York City Area. JAMA. 2020. doi:10.1001/jama.2020.6775

17. CDC Weekly C, The Novel Coronavirus Pneumonia Emergency Response Epidemiology Team. The Epidemiological Characteristics of an Outbreak of 2019 Novel Coronavirus Diseases (COVID-19) - China, 2020. China CDC Weekly. 2020;2: 113-122. doi:10.46234/ccdcw2020.032

18. Docherty AB, Harrison EM, Green CA, Hardwick HE, Pius R, Norman L, et al. Features of 20133 UK patients in hospital with covid-19 using the ISARIC WHO Clinical Characterisation Protocol: prospective observational cohort study. BMJ. 2020;369: m1985. doi:10.1136/bmj.m1985

19. Iaccarino G, Grassi G, Borghi C, Ferri C, Salvetti M, Volpe M, et al. Age and Multimorbidity Predict Death Among COVID-19 Patients: Results of the SARS-RAS Study of the Italian Society of Hypertension. Hypertension. 2020;76: 366-372. doi:10.1161/HYPERTENSIONAHA.120.15324

20. Imam Z, Odish F, Gill I, O'Connor D, Armstrong J, Vanood A, et al. Older age and comorbidity are independent mortality predictors in a large cohort of 1305 COVID-19 patients in Michigan, United States. J Intern Med. 2020. doi:10.1111/joim.13119

21. Christensen DM, Strange JE, Gislason G, Torp-Pedersen C, Gerds T, Fosbøl E, et al. Charlson Comorbidity Index Score and Risk of Severe Outcome and Death in Danish COVID-19 Patients. J Gen Intern Med. 2020. doi:10.1007/s11606-020-05991-z

22. Xie J, Tong Z, Guan X, Du B, Qiu H, Slutsky AS. Critical care crisis and some recommendations during the COVID-19 epidemic in China. Intensive Care Med. 2020;46: 837-840. doi:10.1007/s00134-020-05979-7 
23. Shah S, Majmudar K, Stein A, Gupta N, Suppes S, Karamanis M, et al. Novel Use of Home Pulse Oximetry Monitoring in COVID-19 Patients Discharged From the Emergency Department Identifies Need for Hospitalization. Acad Emerg Med. 2020. doi:10.1111/acem.14053

24. Fine MJ, Auble TE, Yealy DM, Hanusa BH, Weissfeld LA, Singer DE, et al. A prediction rule to identify low-risk patients with community-acquired pneumonia. N Engl J Med. 1997;336: 243-250.

doi:10.1056/NEJM199701233360402

25. Kolditz M, Braeken D, Ewig S, Rohde G. Severity Assessment and the Immediate and Long-Term Prognosis in Community-Acquired Pneumonia. Semin Respir Crit Care Med. 2016;37: 886-896. doi:10.1055/s-0036-1592127

26. Wesemann T, Nüllmann H, Pflug MA, Heppner HJ, Pientka L, Thiem U. Pneumonia severity, comorbidity and 1year mortality in predominantly older adults with community-acquired pneumonia: a cohort study. BMC Infect Dis. 2015;15: 2. doi:10.1186/s12879-014-0730-x

27. Bahlis LF, Diogo LP, Kuchenbecker R de S, Fuchs SC. Clinical, epidemiological, and etiological profile of inpatients with community-acquired pneumonia in a public hospital in the interior of Brazil. J Bras Pneumol. 2018;44: 261-266. doi:10.1590/S1806-37562017000000434

28. Watanabe Tejada LC, Paje D, Shakeel QL, Uduman AK, Vahia A, Cabrera. R. Effect of comorbidities on clinical outcomes in low-risk curb-65 patients. J Gen Intern Med. 2013;28: S67. Available:

https://link.springer.com/article/10.10072Fs11606-013-2436-y

29. Petrilli CM, Jones SA, Yang J, Rajagopalan H, O’Donnell L, Chernyak Y, et al. Factors associated with hospital admission and critical illness among 5279 people with coronavirus disease 2019 in New York City: prospective cohort study. BMJ. 2020;369: m1966. doi:10.1136/bmj.m1966

30. Knight SR, Ho A, Pius R, Buchan I, Carson G, Drake TM, et al. Risk stratification of patients admitted to hospital with covid-19 using the ISARIC WHO Clinical Characterisation Protocol: development and validation of the 4C Mortality Score. BMJ. 2020;370: m3339. doi:10.1136/bmj.m3339

31. Bernabeu-Wittel M, Ternero-Vega JE, Díaz-Jiménez P, Conde-Guzmán C, Nieto-Martín MD, Moreno-Gaviño L, et al. Death risk stratification in elderly patients with covid-19. A comparative cohort study in nursing homes outbreaks. Arch Gerontol Geriatr. 2020;91: 104240. doi:10.1016/j.archger.2020.104240

32. Berenguer J, Ryan P, Rodríguez-Baño J, Jarrín I, Carratalà J, Pachón J, et al. Characteristics and predictors of death among 4,035 consecutively hospitalized patients with COVID-19 in Spain. Clin Microbiol Infect. 2020. doi:10.1016/j.cmi.2020.07.024

33. Wu Z, McGoogan JM. Characteristics of and Important Lessons From the Coronavirus Disease 2019 (COVID19) Outbreak in China: Summary of a Report of 72314 Cases From the Chinese Center for Disease Control and Prevention. JAMA. 2020. doi:10.1001/jama.2020.2648

34. Colombi D, Bodini FC, Petrini M, Maffi G, Morelli N, Milanese G, et al. Well-aerated Lung on Admitting Chest CT to Predict Adverse Outcome in COVID-19 Pneumonia. Radiology. 2020;296: E86-E96. doi:10.1148/radiol.2020201433

35. Ji D, Zhang D, Xu J, Chen Z, Yang T, Zhao P, et al. Prediction for Progression Risk in Patients with COVID-19 Pneumonia: the CALL Score. Clin Infect Dis. 2020. doi:10.1093/cid/ciaa414

36. Singh K, Valley TS, Tang S, Li BY, Kamran F, Sjoding MW, et al. Validating a Widely Implemented Deterioration Index Model Among Hospitalized COVID-19 Patients. medRxiv. 2020. doi:10.1101/2020.04.24.20079012

37. Bai X, Fang C, Zhou Y, Bai S, Liu Z, Chen Q, et al. Predicting COVID-19 malignant progression with Al techniques. medRxiv. 2020; 2020.03.20.20037325. doi:10.1101/2020.03.20.20037325 
38. Huang H, Cai S, Li Y, Li Y, Fan Y, Li L, et al. Prognostic factors for COVID-19 pneumonia progression to severe symptom based on the earlier clinical features: a retrospective analysis. medRxiv. 2020; 2020.03.28.20045989. doi:10.1101/2020.03.28.20045989

39. Carr E, Bendayan R, O'Gallagher K, Bean D, Dobson R. Supplementing the National Early Warning Score (NEWS2) for anticipating early deterioration among patients with COVID-19 infection. 2020 [cited 27 Aug 2020]. doi:10.1101/2020.04.24.20078006

40. Gong J, Ou J, Qiu X, Jie Y, Chen Y, Yuan L, et al. A Tool to Early Predict Severe 2019-Novel Coronavirus Pneumonia (COVID-19) : A Multicenter Study using the Risk Nomogram in Wuhan and Guangdong, China. medRxiv. 2020; 2020.03.17.20037515. doi:10.1101/2020.03.17.20037515

41. Guo Y, Liu Y, Lu J, Fan R, Zhang F, Yin X, et al. Development and validation of an early warning score (EWAS) for predicting clinical deterioration in patients with coronavirus disease 2019. Infectious Diseases (except HIV/AIDS). medRxiv; 2020. p. 2020.04.17.20064691. doi:10.1101/2020.04.17.20064691

42. Smith G, Nielsen M. ABC of intensive care. Criteria for admission. BMJ. 1999;318: 1544-1547. doi:10.1136/bmj.318.7197.1544

43. Metlay JP, Waterer GW, Long AC, Anzueto A, Brozek J, Crothers K, et al. Diagnosis and Treatment of Adults with Community-acquired Pneumonia. An Official Clinical Practice Guideline of the American Thoracic Society and Infectious Diseases Society of America. Am J Respir Crit Care Med. 2019;200: e45-e67. doi:10.1164/rccm.201908-1581ST

44. Christian MD, Joynt GM, Hick JL, Colvin J, Danis M, Sprung CL, et al. Chapter 7. Critical care triage. Recommendations and standard operating procedures for intensive care unit and hospital preparations for an influenza epidemic or mass disaster. Intensive Care Med. 2010;36 Suppl 1: S55-64. doi:10.1007/s00134-0101765-0

45. Ewig S, Birkner N, Strauss R, Schaefer E, Pauletzki J, Bischoff H, et al. New perspectives on community-acquired pneumonia in 388406 patients. Results from a nationwide mandatory performance measurement programme in healthcare quality. Thorax. 2009;64: 1062-1069. doi:10.1136/thx.2008.109785

46. Das A, Mishra S, Gopalan SS. Predicting community mortality risk due to CoVID-19 using machine learning and development of a prediction tool. Health Informatics. medRxiv; 2020. doi:10.1101/2020.04.27.20081794

47. Zeng L, Li J, Liao M, Hua R, Huang P, Zhang M, et al. Risk assessment of progression to severe conditions for patients with COVID-19 pneumonia: a single-center retrospective study. medRxiv. 2020; 2020.03.25.20043166. doi:10.1101/2020.03.25.20043166

48. Giacomelli A, Ridolfo AL, Milazzo L, Oreni L, Bernacchia D, Siano M, et al. 30-day mortality in patients hospitalized with COVID-19 during the first wave of the Italian epidemic: A prospective cohort study. Pharmacol Res. 2020;158: 104931. doi:10.1016/j.phrs.2020.104931

49. Ahmad OB, Boschi-Pinto C, Lopez AD, Murray CJL, Lozano R, Inoue M, et al. Age standardization of rates: a new WHO standard. World Health Organization. 2001;9. Available: https://www.who.int/healthinfo/paper31.pdf

50. Rao C, Doi S, Williams G. Enhanced comparisons of COVID-19 mortality across populations. Epidemiology. medRxiv; 2020. doi:10.1101/2020.06.14.20131318

51. Ocaña-Riola R. Common errors in disease mapping. Geospat Health. 2010;4: 139-154. doi:10.4081/gh.2010.196

52. Guan W-J, Ni Z-Y, Hu Y, Liang W-H, Ou C-Q, He J-X, et al. Clinical Characteristics of Coronavirus Disease 2019 in China. N Engl J Med. 2020;382: 1708-1720. doi:10.1056/NEJMoa2002032 
53. Bottle A, Jarman B, Aylin P. Strengths and weaknesses of hospital standardised mortality ratios. BMJ. 2010;342: c7116. doi:10.1136/bmj.c7116

\section{Figures}

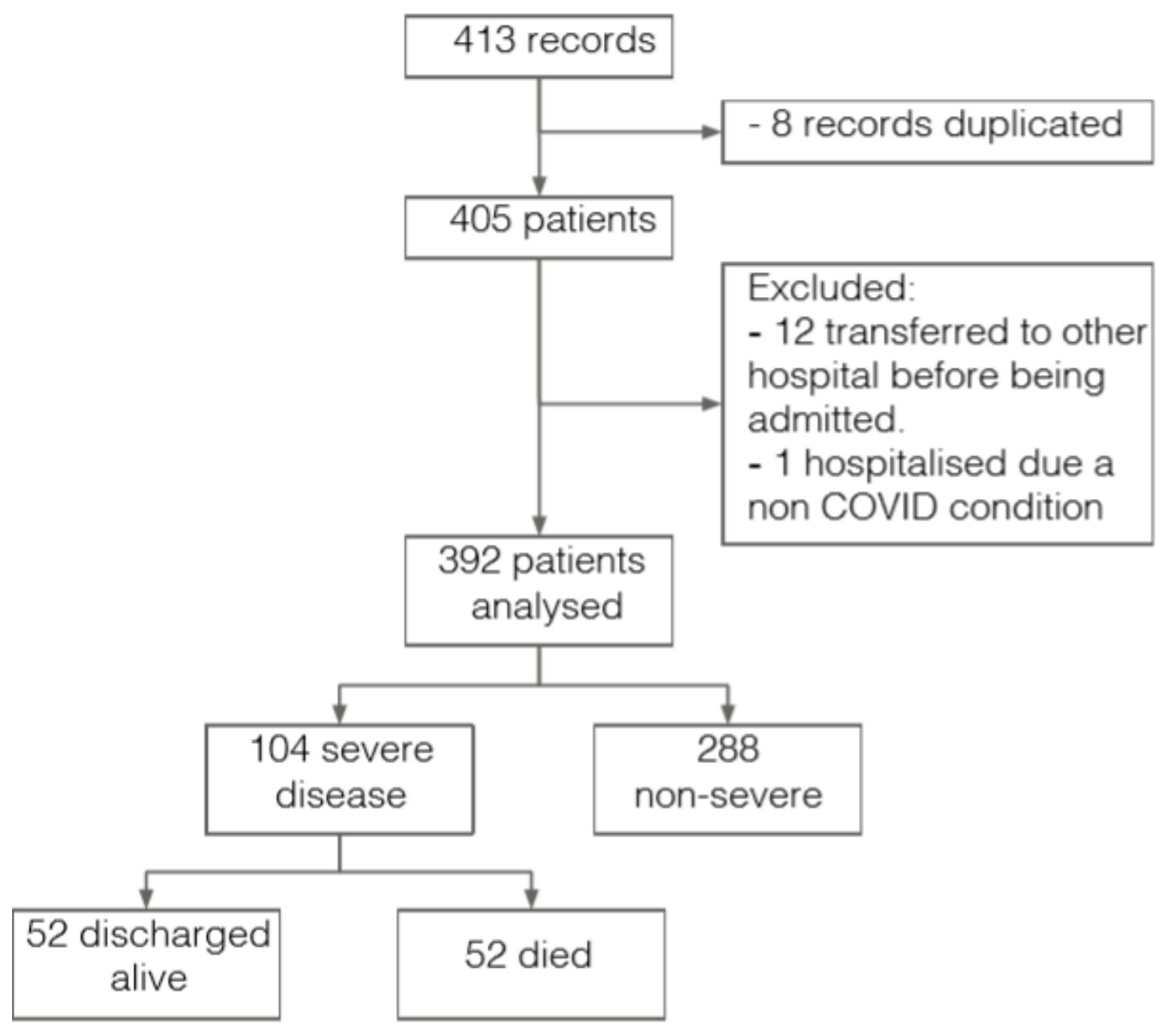

Figure 1

Participants flowchart. 


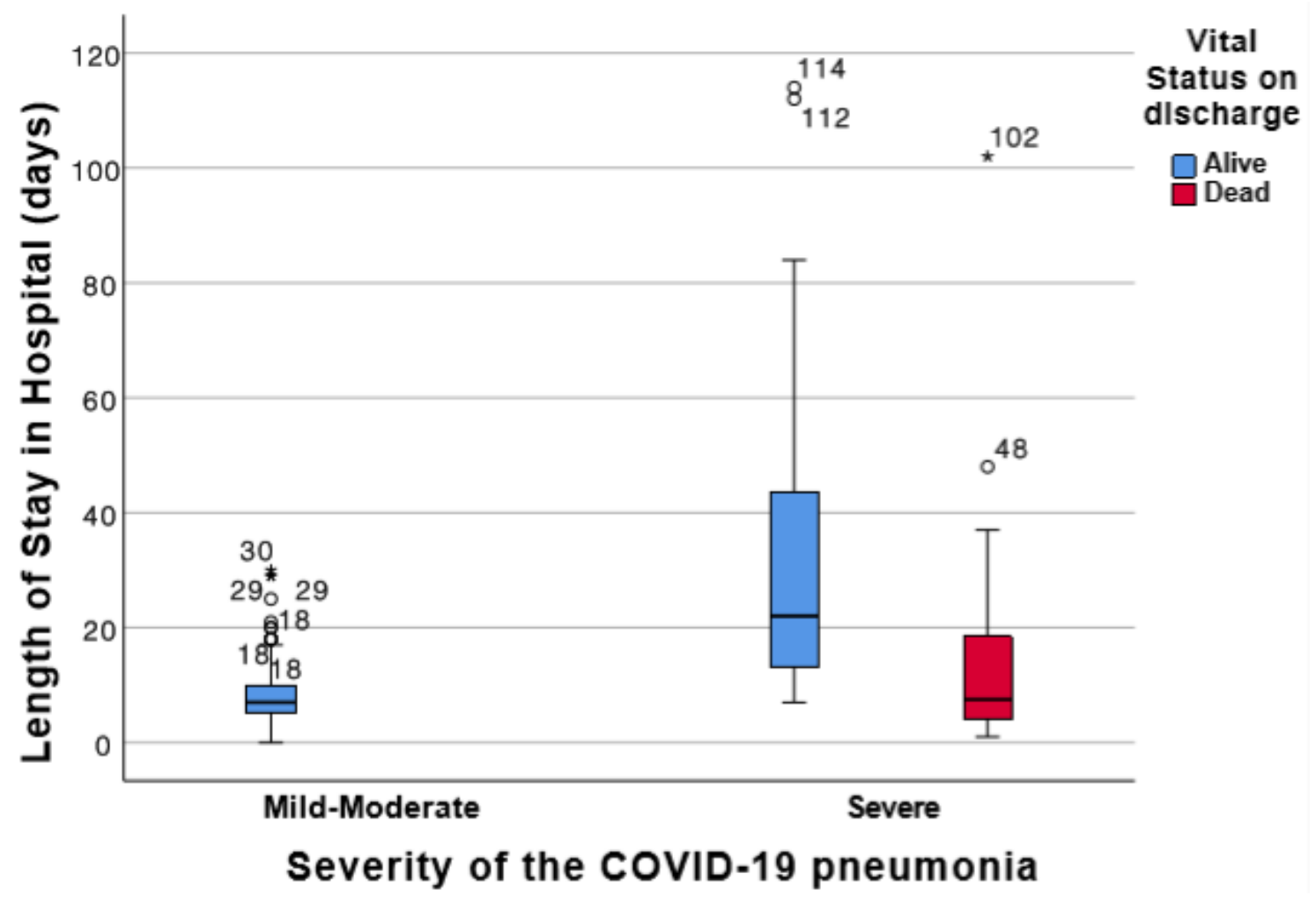

Figure 2

Relationship between length of stay in hospital - severity-vital status on discharge. Box plot showing the length of stay in hospital, according to the severity of the disease and status at discharge. Numbers in the graph area indicate length of stay. 


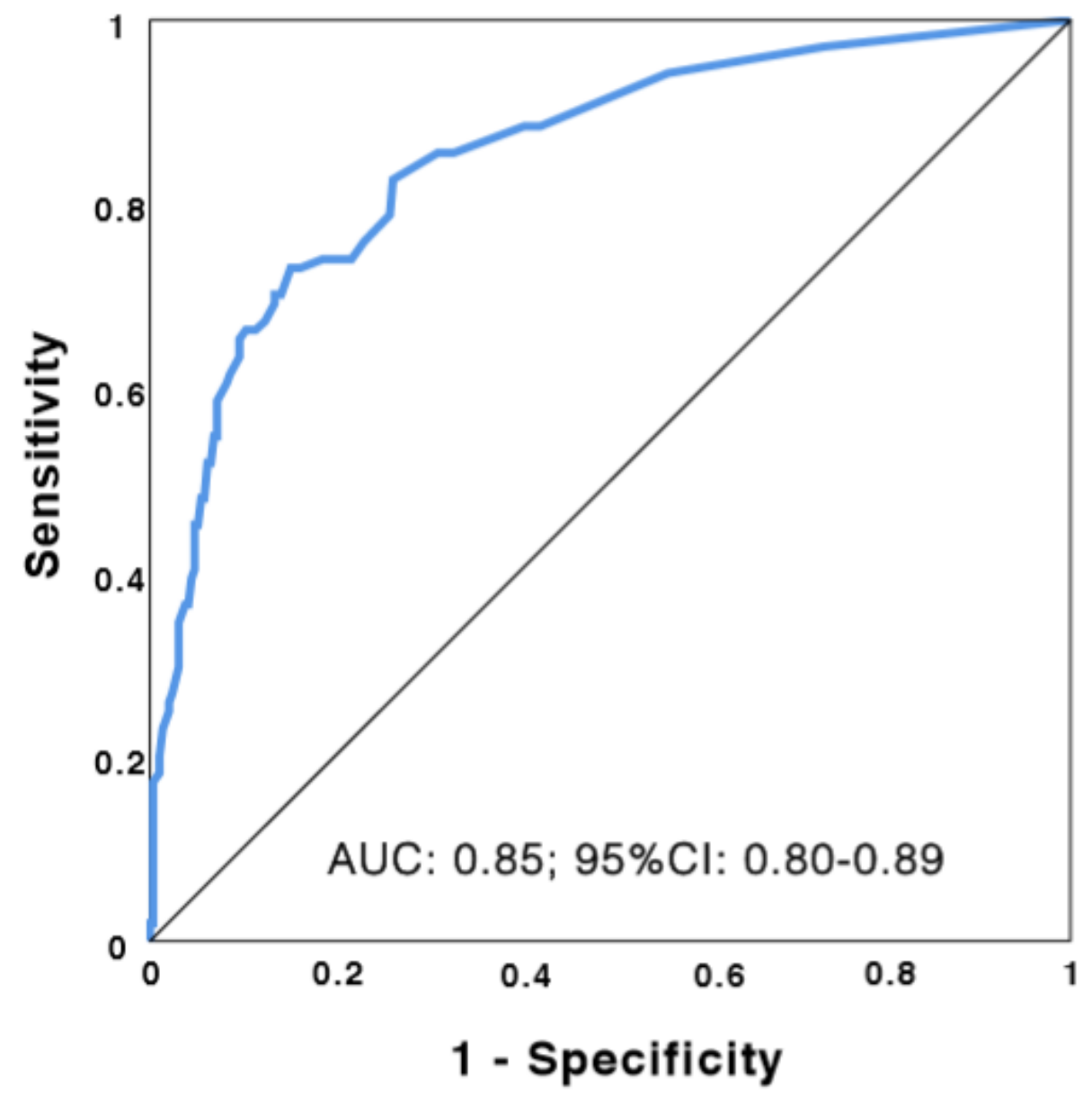

Figure 3

ROC curve of the severe disease prediction model. 


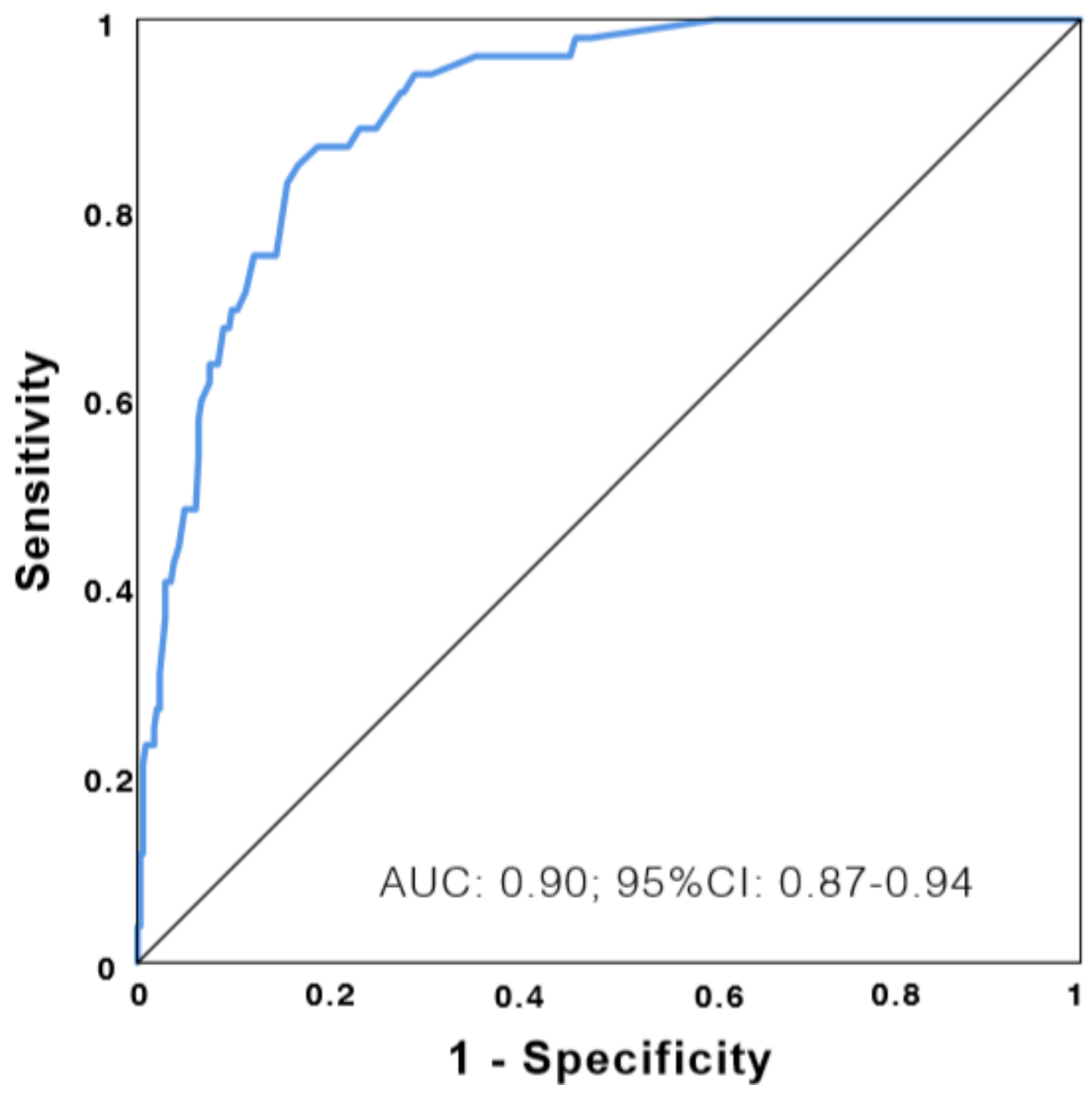

Figure 4

ROC curve of the predictive model of mortality. 


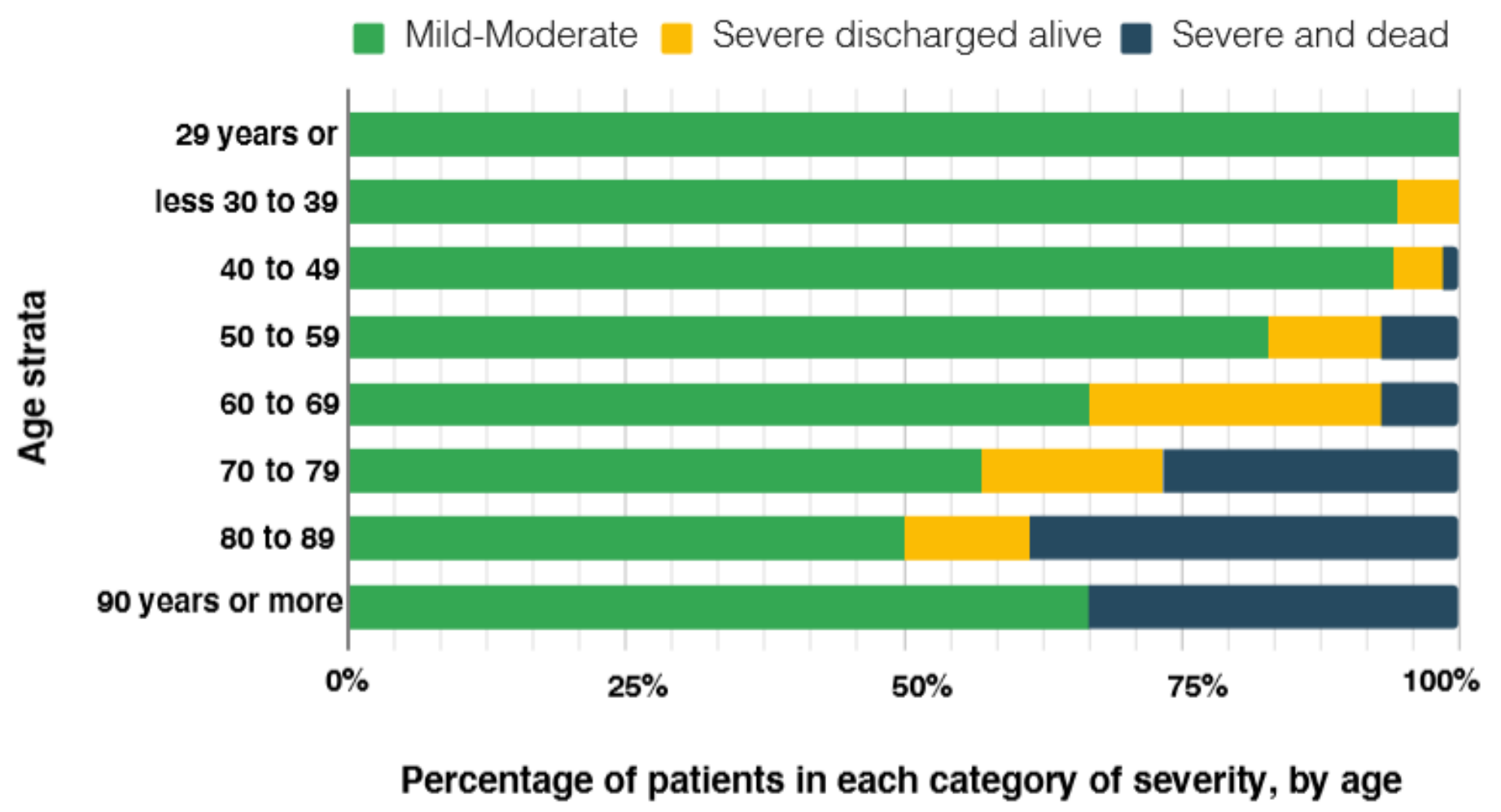

Figure 5

Percentage of severe disease and mortality, by age strata. Total patients in each decade: 29 years or less: 6 patients; 30-39: 35 patients; 40-49: 68 patients; 50-59: 70 patients; 60-69: 84 patients; 70-79: 79 patients; 80-89: 44 patients; 90 years or older: 6 patients.

\section{Supplementary Files}

This is a list of supplementary files associated with this preprint. Click to download.

- Graphicalabstract.jpg

- SUPPLEMENTARYMATERIAL.pdf

- Additionalfile1.pdf

- Additionalfile2.docx

- Additionalfile3.docx

- Additionalfile4.docx

- Additionalfile5.docx

- Additionalfile6.pdf

- Additionalfile7.docx

- Additionalfile8.pdf

- Additionalfile9.pdf 
- Additionalfile10.pdf

Page $30 / 30$ 